Dear Author,

Please, note that changes made to the HTML content will be added to the article before publication, but are not reflected in this PDF.

Note also that this file should not be used for submitting corrections. 


\title{
Stochastic model predictive control for economic/environmental operation management of microgrids: An experimental case study ${ }^{\text {is }}$
}

\author{
Q1 Alessandra Parisio ${ }^{\mathrm{a}, *}$, Evangelos Rikos ${ }^{\mathrm{b}}$, Luigi Glielmo ${ }^{\mathrm{c}}$ \\ a School of Electrical and Electronic Engineering, The University of Manchester, Manchester, United Kingdom \\ ${ }^{\mathrm{b}}$ Department of PVs and DER Systems, Center for Renewable Energy Sources and Saving (CRES), Pikermi, Athens, Greece \\ c Department of Engineering, Università degli Studi del Sannio, Benevento, Italy
}

\section{A R T I C L E I N F O}

\section{Article history:}

Received 7 February 2015

Received in revised form 1 February 2016 Accepted 10 April 2016

Available online $\mathrm{xxx}$

\section{Keywords:}

Stochastic model predictive control

Two stage stochastic programming

Microgrids

Optimization

Mixed integer linear programming

\begin{abstract}
A B S T R A C T
Microgrids are subsystems of the distribution grid which comprises generation capacities, storage devices and flexible loads, operating as a single controllable system either connected or isolated from the utility grid. In this work, microgrid management system is developed in a stochastic framework. It is seen as a constraint-based system that employs forecasts and stochastic techniques to manage microgrid operations. Uncertainties due to fluctuating demand and generation from renewable energy sources are taken into account and a two-stage stochastic programming approach is applied to efficiently optimize microgrid operations while satisfying a time-varying request and operation constraints. At the first stage, before the realizations of the random variables are known, a decision on the microgrid operations has to be made. At the second stage, after random variables outcomes become known, correction actions must be taken, which have a cost. The proposed approach aims at minimizing the expected cost of correction actions. Mathematically, the stochastic optimization problem is stated as a mixed-integer linear programming problem, which is solved in an efficient way by using commercial solvers. The stochastic problem is incorporated in a model predictive control (MPC) scheme to further compensate the uncertainty through the feedback mechanism. A case study of a microgrid is employed to assess the performance of the on-line optimization-based control strategy and the simulation results are discussed. The method is applied to an experimental microgrid: experimental results show the feasibility and the effectiveness of the proposed approach.
\end{abstract}

(C) 2016 Published by Elsevier Ltd.

\section{Introduction}

Microgrids are integrated energy systems with the possibility of bidirectional power flow, which can operate either in parallel with the grid or in an intentional island mode. A typical microgrid consists of interconnected loads and distributed energy resources (DERs), i.e., distributed generators (DGs), renewable energy resources (RESs) and storage devices [1,2]. Microgrids have also the ability of supplying thermal load with combined heat and power (CHP) capabilities; this would increase the overall system efficiency. The microgrid concept could efficiently support high penetration of RESs, storages and the integration on demand

\footnotetext{
The work was partially funded by the Distributed Energy Resources Research Infrastructures (DERri) and the e-GOTHAM (Sustainable-Smart Grid Open System for the Aggregated Control, Monitoring and Management of Energy) projects.

* Corresponding author. Tel.: +44 7492599594.

E-mail addresses: alessandra.parisio@manchester.ac.uk (A. Parisio), vrikos@cres.gr (E. Rikos),gliemo@unisannio.it (L. Glielmo).
}

response policies in the smart grid [3,2]. This led to the motivation behind using microgrids, which are capable of managing and coordinating DERs and loads in a more decentralized way reducing the need for the centralized coordination and management [4]. The growing need of reducing carbon emissions makes the concept of microgrid even more attractive.

Thus, in the smart grid scenario, new modeling requirements are needed, e.g. storage modeling must be incorporated into the operation planning problem in order to coordinate storage use with RES generation and energy prices, and address the complexity of the charging/discharging schedule [5]. It is important to notice that there are no current modeling tools including flexible loads and energy storage modeling in a smart grid environment, which also take the inherent uncertainty sources into account [6]. The modeling capabilities and the computational advances of Mixed Integer Problem (MIP) algorithms, have led several Independent System Operators (ISOs) and Regional Transmission Organizations (RTOs) to implement MIP-based solution methods in order to find a better solution to solve day-ahead and real-time market problems [7]; however, not solving unit commitment problems to complete 
optimality can cause several issues, such as incorrect energy prices (generator payoff deviations) and surplus issues [8].

The main objectives from an energy and control prospective are to coordinate various DGs, RESs, storages and loads in order to determine the optimal energy scheduling depending on running costs, thermal and electrical energy requirements and consumers preferences; the main challenges are then represented by flexible loads, which account for consumers preferences, and uncertainties due to RESs and loads [9].

In this paper we tackle the optimal economic/environmental operation management of a microgrid under supply and load uncertainties. This problem aims at achieving the optimal tradeoff between the overall microgrid operating costs and emissions to meet load demand of a certain period (typically one day) while satisfying complex operational constraints.

Literature review. Though there is already a vast literature on microgrids energy management system, there are still many research and development needs associated to microgrids, among these one of the most crucial elements is finding the best operational strategy under uncertainty.

Several models have been proposed for microgrids, some of them solving also the microgrid environmental/economic optimization problem (e.g., [10-13]).

Typically the proposed approaches either are computationally intensive and/or heuristics or decomposition techniques are applied; further either the optimization problem stays nonlinear or other important features, such as minimum up and down times and demand side programs, are neglected, e.g. see also [14-20].

In a stochastic framework, multistage stochastic programming, in particular with a two-stage form, has had several applications in energy models. Literature on optimization under uncertain conditions indicates that stochastic programming is considered as an appropriate approach for handling uncertainties (see [21,22] and references therein). Stochastic approaches have been proposed in literature for smart grids and microgrids, with the goal of minimizing running costs and/or emissions, e.g., [23-27]. Multi-objective approaches employ $\varepsilon$-constraint technique and lexicographic optimization to solve the microgrid scheduling problem $[28,29]$. Simulation results show that generally the stochastic decisions outperform the deterministic decisions.

Uncertainties in load forecasts and RES generation are generally taken into account; typically the microgrid optimization problem is formulated as a mixed integer nonlinear problem and decomposition techniques or heuristic-based approaches such as evolution algorithms and particles swarm optimization are proposed [30,28,31-39]. In particular, in [38] a robust agent-based microgrid energy management framework is proposed, which takes the probability of occurrence of some specific uncertain events, e.g., failures of electrical lines and electricity demand and price peaks, into account.

Recently, model predictive control (MPC) has drawn the attention of the power system community [40]. In power system literature, an MPC method, called rolling horizon, has been commonly proposed to solve the unit commitment problem with wind power generation (e.g., see [41]).

Several works can be found in the literature that addresses MPC for optimal dispatch in power systems, e.g., [42-47]. Several authors showed the advantages of applying MPC to dynamic economic dispatch in a stochastic or in an environmental framework $[48,49]$. Some authors have shown that a stochastic MPC is a promising approach $[50,49]$.

As before, in the solution methods proposed above, the optimization problem stays nonlinear and/or heuristics or decomposition techniques are applied; important microgrid features are in general neglected such as storage dynamics, grid interaction, generators operative constraints.
An integrated optimization-based framework for microgrid operational planning has not yet been proposed, which considers the inherent uncertainty sources, all kinds of distributed energy resources technologies (DGs, RESs, energy storages and flexible loads), as well as detailed system features and operating constraints, from both an economic and environmental viewpoint.

We would like to remark that, when storage elements are considered, generally the proposed storage modeling does not rule out the possibility that the optimal mathematical solution contemplates simultaneous charging and discharging of the storage, a physically unrealizable policy. Such outcome may occur as the mathematical consequence of unpredicted RES-generated power surplus, bounds on the exchanged power with the utility grid, and costs of the storage level. This issue has been never discussed in the corresponding studies. Similarly the interaction with the utility grid should be modeled so as to prevent solutions contemplating simultaneous selling and purchasing under certain market circumstances.

Main assumptions and contributions. Since microgrid control requirements involve different control approaches and different time scales, the typical control hierarchy of a microgrid (e.g., IEC/ISO62264 standard) requires a centralized, high level controller on the top of the hierarchy and local controllers associated with each DER and load unit $[9,51]$. The high level controller deals with longer time scales and aims at generating suitable set-points for DERs and flexible load units so that optimized power dispatch will be performed and a given demand is met. The local controller embedded in a DER is mainly responsible for voltage/frequency and active/reactive power control in order to ensure that the power reference values are tracked, keeping voltage stability and power quality (which has higher priority) [9].

The high level controller is very weakly dependent on the transient behavior of the fast dynamics. Then a steady state assumption for microgrid components can be safely made without much loss of accuracy. A further assumption of our approach is that the Microgrid Operator is the unique centralized entity in charge of microgrid management.

In this paper we develop an integrated optimization-based framework for microgrid economical/environmental operational planning in a stochastic framework, considering both thermal and electrical energy demand. We propose the use of MPC in combination with mixed integer linear programming (MILP) [52,53] and two-stage stochastic programming [54]; we will refer the proposed novel control scheme for microgrid management as MG-SMPC.

By taking the environmental concern into account, the microgrid operation optimization problem can be formulated as a multi-objective optimization (MOO) problem with conflicting objectives (operative costs and emissions), for which a set of optimal solutions (Pareto-optimal set) and the corresponding objective function values (Pareto curve) are computed. In this paper, the weighted min-max method is applied to compute Pareto optimal points; this approach provides both necessary and sufficient conditions for Pareto optimality [55]. The desired number of Paretooptimal (non-dominated) solutions can be obtained by varying the weights on the objective functions.

In our problem formulation, we strove to include as many details as possible and maintain the microgrid stochastic optimization problem solvable by standard algorithms without resorting to any decomposition techniques or heuristics; this leads to improvements in solution quality and computational burden [56,57].

To guarantee a correct behavior for storage and grid interaction (e.g., non-simultaneous charging and discharging), we utilize the approach described in [58] and use the Mixed Logical Dynamical (MLD) framework. We would like to remark that, in the proposed problem formulation, only generators fuel consumption and emission functions are approximated in case they must be expressed 
Table 1

Parameters.

\begin{tabular}{ll}
\hline$N_{g}, N_{\text {chp }}$ & Number of DGs, whose $N_{\text {chp }}$ are CHP systems \\
$N_{l}, N_{q}, N_{c}$ & Number of (electrical and thermal) critical loads and flexible loads \\
$C^{\mathrm{DG}}(P)$ & Fuel consumption cost curve of a DG [€] \\
$E(P)$ & Emission function of a DG [kg] \\
$O M$ & Operating and maintenance cost of a DG [€/kWh] \\
$R_{\max }$ & Ramp up limit of a DG [kW/h] \\
$T$ & Prediction horizon [fraction of hour] \\
$\mathrm{PHR}$ & Power to heat ratio of a CHP system \\
$T^{\mathrm{up}}, T^{\text {down }}$ & Minimum up and down time of a DG [fraction of hour] \\
$\alpha^{\mathrm{sb}}$ & Storage self-consumption per kWh \\
$x_{\min }^{b}, x_{\max }^{b}$ & Minimum, maximum storage energy level [kWh] \\
$C_{\max }^{b}$ & Storage power limit [kW] \\
$T^{g}$ & Maximum grid interconnection power limit [kW] \\
$P_{\min }, P_{\max }$ & Minimum, maximum power level of a DG [kW] \\
$\eta^{c}, \eta^{d}$ & Storage charging and discharging efficiencies \\
$\beta_{\min }, \beta_{\max }$ & Minimum, maximum curtailment of flexible loads \\
$c^{\mathrm{SU}}, c^{\mathrm{SD}}$ & Start-up, shut-down costs of a DG [€] \\
$D^{c}$ & Preferred power level of a flexible load [kW] \\
$\rho_{c}$ & Penalty weight on curtailments \\
$q^{+}, q^{-}$ & Costs on recourse actions [€/kWh] \\
\hline
\end{tabular}

Table 2

Forecasts.

Pres Sum of power production from RES [kW]

$c^{P}, c^{S} \quad$ Purchasing, selling energy prices $[€ / \mathrm{kWh}]$

Table 3

Decision and logical variables.

\begin{tabular}{ll}
\hline$\delta$ & Off (0)/on (1) state of a DG \\
$\delta^{b}$ & Discharging (0)/charging (1) mode of the storage unit \\
$\delta^{g}$ & Exporting (0)/importing (1) mode to/from the utility grid \\
$P, Q$ & Electrical $(P)$ and thermal $(Q)$ power level of a DG [kW] \\
$P^{b}$ & Power exchanged (positive for charging) with the storage \\
& unit [kW] \\
$P^{g}$ & Importing (positive)/exporting (negative) power level \\
& from/to the utility grid [kW] \\
$C^{g}$ & Cost of power exchanged with the utility grid [€] \\
$x^{b}$ & Stored energy level [kWh] \\
$\beta$ & Curtailed power faction \\
$\xi^{-}, \xi^{+}$ & Recourse variables associated to electrical loads [kW] \\
$\xi_{q}^{-}, \xi_{q}^{+}$ & Recourse variables associated to thermal loads [kW]
\end{tabular}

tion 2 describes the microgrid modeling, while Section 3 presents to the optimization model are presented in Section 4. Section 5 reports and discusses numerical and experimental results. Section 6 ends the manuscript with a summary of our conclusions and indications of future research directions.

\subsection{Nomenclature}

The parameters, the forecasts and the decision variables used in the proposed formulation are described respectively in Tables $1-3$, where, for simplicity, the subscripts $i$ and $h$ when referring to the $i$ th generating unit and the $h$ th load are omitted. The fuel consumption
$D, D^{Q} \quad$ Electrical $(D)$ and thermal $\left(D^{Q}\right)$ power required by a critical load [kW] the novel stochastic MPC control scheme for microgrids. Extensions cost for a DG unit is traditionally assumed to be a quadratic function of the generated power of the form $C^{D G}(P)=a_{1} P^{2}+a_{2} P+a_{3}$. bold.

In the following sections, vectors and matrices are denoted in

\section{Microgrid modeling}

In this section we describe the key features of the microgrid system, which comprises continuous-time dynamics of energy flows and storage units, and on/off status of microgrid units. The modeling set up is developed with the goal of maintaining the problem tractable (see [60] for further details).

In this work, the proposed microgrid modeling also includes the supply of thermal load with combined heat and power CHP capabilities.

We remark that, due to constant sampling time $\Delta T=t_{k+1}-t_{k}$, there exists a constant ratio between energy and power at each interval.

\subsection{Loads}

As mentioned in [60], we consider two types of loads: (i) critical loads, i.e. demand levels related to essential processes that must be always met; (ii) flexible loads, i.e. loads that can be reduced or shed during supply constraints or emergency situations. A continuousvalued variable, $0 \leq \beta_{h}(k) \leq 1$, associated to each flexible load $h$ and to each sampling time $k$ is defined. This variable represents the percentage of preferred power level to be curtailed at time $k$.

\subsection{Interaction with the utility grid}

By employing the MLD framework, we model the possibility either to purchase or to sell energy from/to the utility grid by introducing a binary variable $\delta^{g}(k)$ and an auxiliary variable $C^{g}(k)$ such that:

$C^{g}(k)= \begin{cases}c^{P}(k) P^{g}(k) & \text { if } \delta^{g}(k)=1, \\ c^{S}(k) P^{g}(k) & \text { otherwise. }\end{cases}$

Again, we express the 'if ... then' conditions as mixed integer linear inequalities. Then, the purchasing/selling microgrid behavior can be compactly expressed by the following mixed integer linear inequalities:

$\mathrm{E}_{\mathbf{1}}{ }^{g} \delta^{g}(k)+\mathrm{E}_{\mathbf{2}}{ }^{g} C^{g}(k) \leq \mathrm{E}_{\mathbf{3}}{ }^{g}(k) P^{g}(k)+\mathrm{E}_{\mathbf{4}}{ }^{g}$.

The column vectors $\mathbf{E}_{1}{ }^{g}, \mathbf{E}_{2}{ }^{g}, \mathbf{E}_{3}{ }^{g}(k), \mathbf{E}_{4}{ }^{g}$ are given in Appendix $A$. The interested reader can find further details in [60]. The vector $\mathbf{E}_{3} g(k)$ is generally time-varying due to the time-varying energy prices.

\subsection{Storage dynamics}

The following discrete time model of a storage unit is considered:

$x^{b}(k+1)=\alpha^{\mathrm{sb}} x^{b}(k)+\eta P^{b}(k)$,

where

$\eta=\left\{\begin{array}{lll}\eta^{c}, & \text { if } P^{b}(k)>0 & \text { (charging mode) } \\ \eta^{d}, & \text { otherwise } & \text { (discharging mode) }\end{array}\right\}$

Typically $\alpha^{\mathrm{sb}}<1, \eta^{c}<1$ and $\eta^{d}=1 / \eta^{c}$ account for energy losses.

By using the MLD framework described in [58], a binary variable $\delta^{b}(k)$ and an auxiliary variable $z^{b}(k)=\delta^{b}(k) P^{b}(k)$ are introduced to model the logical conditions provided in (4); the storage dynamics 
$x^{b}(k+1)=\alpha^{\mathrm{sb}} x^{b}(k)-\left(\eta^{d}-\eta^{c}\right) z^{b}(k)+\eta^{d} P^{b}(k)$,

subject to $\mathrm{E}_{1}{ }^{b} \delta^{b}(k)+\mathrm{E}_{2}{ }^{b} z^{b}(k) \leq \mathrm{E}_{3}{ }^{b} P^{b}(k)+\mathrm{E}_{4}{ }^{b}$,

where the column vectors $\mathbf{E}_{1}{ }^{b}, \mathbf{E}_{2}{ }^{b}, \mathbf{E}_{3}{ }^{b}, \mathbf{E}_{4}{ }^{b}$ are given in Appendix A. The interested reader can find further details in [60].

\subsection{Electrical and thermal balance}

The balance between electrical energy production and consumption must be met at each time $k$; this is expressed as follows:

$$
\begin{aligned}
P^{b}(k)= & \sum_{i=1}^{N_{g}} P_{i}(k)+P^{\mathrm{res}}(k)+P^{g}(k)-\sum_{j=1}^{N_{l}} D_{j}(k) \\
& -\sum_{h=1}^{N_{c}}\left[1-\beta_{h}(k)\right] D_{h}^{c}(k) .
\end{aligned}
$$

As mentioned in the introduction, our microgrid modeling also includes the ability of supplying thermal load with CHP capabilities. The total efficiency of a CHP system is defined as the sum of the electrical and thermal energy outputs divided by the total energy of the fuel consumed. Another important concept related to CHP system efficiency is the power-to-heat ratio (PHR), which indicates the proportion of power (electrical or mechanical energy) to heat energy (steam or hot water) produced in the CHP system [62,63].

The following linear constraints must be included in the optimization problem to consider thermal energy in microgrids:

$\sum_{i=1}^{N_{\text {chp }}} Q_{i}(k)=\sum_{j=1}^{N_{q}} D_{j}^{Q}(k)$,

$P_{i}(k)=\operatorname{PHR}_{i} Q_{i}(k), \quad i=1, \ldots, N_{\text {chp }}$.

We remark that gas and waste heat boilers can be easily included in the left-hand side of the thermal balance constraints and in the objective function to account for their running costs.

In order to clearly describe the two-stage stochastic approach in Section 3.2, it is more convenient to rewrite the electrical and thermal power balance equations in a compact form. Denote by $\boldsymbol{u}(k)$ the vector collecting all decision variables and by $\boldsymbol{w}(k)$ the vector containing all disturbances

$\boldsymbol{u}(k)=\left[\begin{array}{llllllll}P^{b}(k) & \boldsymbol{P}^{\prime}(k) & P^{g}(k) & \boldsymbol{\beta}^{\prime}(k) & \boldsymbol{Q}^{\prime}(k) & \boldsymbol{\delta}^{\prime}(k) & \delta^{b}(k) & \delta^{g}(k)\end{array}\right]^{\prime}$,

$\boldsymbol{w}(k)=\left[\begin{array}{llll}P^{\mathrm{res}}(k) & \boldsymbol{D}^{\prime}(k) & \boldsymbol{D}^{c^{\prime}}(k) & \boldsymbol{D}^{Q^{\prime}}(k)\end{array}\right]^{\prime}$,

where $\boldsymbol{u}(k) \in \mathbb{R}^{N_{u}} \times\{0,1\}^{N_{g}+2}, \boldsymbol{w}(k) \in \mathbb{R}^{N_{w}}, N_{u}=N_{g}+1+N_{c}+N_{\text {chp }}$, $N_{w}=1+N_{l}+N_{c}+N_{q}$ and

$\boldsymbol{P}(k):=\left[P_{1}(k) \ldots P_{N_{g}}(k)\right]^{\prime}$,

$\boldsymbol{\beta}(k):=\left[\beta_{1}(k) \ldots \beta_{N_{c}}(k)\right]^{\prime}$,

$\boldsymbol{Q}(k):=\left[Q_{1}(k) \ldots Q_{N_{\text {chp }}}(k)\right]^{\prime}$,

$\delta:=\left[\delta_{1}(k) \ldots \delta_{N_{g}}(k)\right]^{\prime}$,

$\boldsymbol{D}(k):=\left[D_{1}(k) \ldots D_{N_{l}}(k)\right]^{\prime}$,

$\boldsymbol{D}^{\boldsymbol{c}}(k):=\left[D_{1}^{c}(k) \ldots D_{N_{c}}^{c}(k)\right]^{\prime}$,

$\boldsymbol{D}^{\boldsymbol{Q}_{(}}(k):=\left[D_{1}^{Q}(k) \ldots D_{N_{q}}^{Q}(k)\right]^{\prime}$.

By doing so, the electrical and thermal power balance equations can be written as follows

$\boldsymbol{F}(k) \boldsymbol{u}(k)+\boldsymbol{f} \boldsymbol{w}(k)=0$

where $\boldsymbol{F}(k)$ and $\boldsymbol{f}$ are given in Appendix A; $\boldsymbol{F}(k)$ is time-varying since it contains the time-varying preferred energy level for the flexible loads, which is assumed to be known.

\subsection{Generator operating conditions}

The operating constraints, at each sampling time $k$, on the minimum amount of time for which a controllable generation unit must be kept on/off (minimum up/down times) can be expressed by the following mixed integer linear inequalities without resorting to additional variables:

$\delta_{i}(k)-\delta_{i}(k-1) \leq \delta_{i}(\tau) \quad$ (off-on switch),

$\delta_{i}(k-1)-\delta_{i}(k) \leq 1-\delta_{i}(\tau)$ (on-off switch),

with $i=1, \ldots, N_{g}, \tau=k+1, \ldots, \min \left(k+T_{i}^{\mathrm{up}}-1, T\right)$ if the constraints on the minimum up time are considered or $\tau=k+$ $1, \ldots, \min \left(k+T_{i}^{\text {down }}-1, T\right)$ otherwise.

The DG unit start up and shut down behavior are also modeled in order to account for the corresponding costs. For this reason, two auxiliary variables, $S U_{i}(k)$ and $S D_{i}(k)$, are introduced, representing respectively the start up cost and the shut down cost for the $i$ th DG generation unit at time $k$. These auxiliary variables must satisfy the following mixed integer linear constraints:

$S U_{i}(k) \geq c_{i}^{\mathrm{SU}}(k)\left[\delta_{i}(k)-\delta_{i}(k-1)\right]$,

$S D_{i}(k) \geq c_{i}^{\mathrm{SD}}(k)\left[\delta_{i}(k-1)-\delta_{i}(k)\right]$,

$S U_{i}(k) \geq 0$,

$S D_{i}(k) \geq 0$,

with $i=1, \ldots, N_{g}$ (see [64] and references therein). Further details can be found in [60].

\section{Stochastic MPC for microgrid energy management}

This section details the proposed stochastic MPC controller for microgrids. The discussion is divided as follows: (i) in Section 3.1 we recall some basic concept about the two-stage stochastic approach to solve the proposed MPC problem; (ii) in Section 3.2 we formulate the two-stage stochastic problem; (iii) in Section 3.3 we sketch the scenario generation mechanism; (iv) in Section 3.4 we outline the general structure of the stochastic MPC control scheme.

\subsection{Background on two-stage stochastic programming}

This section has been extracted from [54] and provides the basic concepts of stochastic programming with recourse, also known as two-stage stochastic programming. For further background, the reader is referred to [54], and the references therein.

In many practical applications, as in the studied problem, there are several sources of uncertainty in the decision-making process, which affects the decision variables. In the two-stage stochastic programming approach the decision variables are partitioned into two sets. The first-stage variables are those that have to be decided before the actual realization of the uncertain parameters becomes available; once the random events occur, the values of the second stage or recourse variables can be decided. These recourse variables are also interpreted as correction actions as they are used to compensate any infeasibility from the first-stage decisions; thus, violations are accepted, but their costs affect the choice of the first stage variables. The objective is to choose the first-stage variables 
in order to minimize the sum of first-stage costs and the expected value of the random second stage or recourse costs. In the twostage model, the non-anticipativity constraints, stating that the first-stage decisions do not depend on the random variables realizations, are implicitly modeled by not having first-stage decision vectors depending on the random vector.

A two-stage linear stochastic program with simple continuous recourse can be stated as follows:

$$
\min \boldsymbol{c}^{\prime} \boldsymbol{u}+\mathcal{Q}(\boldsymbol{u})
$$

s.t.

$$
\begin{aligned}
& \boldsymbol{A} \boldsymbol{u}=\boldsymbol{b} \\
& \boldsymbol{u} \in \mathcal{U},
\end{aligned}
$$

where $\boldsymbol{u} \in \mathbb{R}^{n}$ is the first stage decision vector, $\mathcal{U}$ is the first stage constraints set, $\boldsymbol{A} \in \mathbb{R}^{s \times n}, \boldsymbol{b} \in \mathbb{R}^{s}, \boldsymbol{\omega} \in \mathbb{R}^{r}$ is the random vector with probability distribution $P_{\boldsymbol{\omega}}$ (assumed being independent from $\boldsymbol{u}$ ) and $\mathcal{Q}(\boldsymbol{u})=\boldsymbol{E}_{\boldsymbol{\omega}}[\Phi(\boldsymbol{u}, \boldsymbol{\omega})]$ is the expected recourse, where $\Phi(\boldsymbol{u}, \boldsymbol{\omega})$ is the second-stage function:

$$
\begin{aligned}
& \Phi(\boldsymbol{u}, \boldsymbol{\omega})=\min \left(\mathbf{q}^{+} \zeta^{+}+\boldsymbol{q}^{-} \zeta^{-}\right) \\
& \text {s.t. } \\
& \quad \zeta^{+} \geq \boldsymbol{H u}-\boldsymbol{\omega} \\
& \zeta^{-} \geq-(\boldsymbol{H u}-\boldsymbol{\omega}) \\
& \zeta^{+}, \zeta^{-} \geq 0,
\end{aligned}
$$

where the inequalities are meant componentwise, $\boldsymbol{q}^{+}$and $\boldsymbol{q}^{-}$are penalty coefficient row vectors of appropriate size and $\zeta^{+}$and $\zeta^{-}$ are the recourse vectors. The random constraints are denoted by the compact form $\mathbf{H u}-\boldsymbol{\omega}=0$.

By introducing $\boldsymbol{y}:=\boldsymbol{H} \boldsymbol{u}$ and considering $P_{\boldsymbol{\omega}}$ as a finite discrete probability distribution with the marginal distribution of $\omega_{i}$ given by $p_{i j}=P_{\omega}\left(\omega_{i}=\hat{\omega}_{i j}\right), j=1, \ldots S_{i}$ and $i=1, \ldots, r$ the $i$ th component, it is straightforward rewriting the problem (11) as the following equivalent deterministic linear problem:

$$
\min \boldsymbol{c}^{\prime} \boldsymbol{u}+\sum_{i=1}^{r} \sum_{j=1}^{S_{i}} p_{i j}\left(q_{i}^{+} \zeta_{i j}^{+}+q_{i}^{-} \zeta_{i j}^{-}\right)
$$

s.t.

$$
\begin{aligned}
& \boldsymbol{A u}=\boldsymbol{b} \\
& \zeta_{i j}^{+} \geq y_{i}-\hat{\omega}_{i j} \\
& \zeta_{i j}^{-} \geq-\left(y_{i}-\hat{\omega}_{i j}\right) \\
& \zeta_{i j}^{+}, \zeta_{i j}^{-} \geq 0 \\
& \boldsymbol{u} \in \mathcal{U} .
\end{aligned}
$$

Further, Problem (11) is said to have complete recourse [54], i.e., the feasible set of the second-stage problem stays non-empty since it is always possible to respond to any possible disturbance realizations in a reasonable time (reasonable with respect to the sampling period).

\subsection{Two-stage stochastic problem formulation}

In this section, the two-stage stochastic problem for microgrid economic/environmental operation management is formulated. The conflicting objectives considered in this problem are running costs and emissions.

\subsubsection{Cost functions}

Microgrid running costs include costs associated with energy production and start-up and shut-down decisions, along with possible profits and curtailment penalties.
Microgrid operating costs. The microgrid running cost at time step $k$ is denoted by $V_{r}(\boldsymbol{u}(k))$ and is given by

$$
\begin{aligned}
V_{r}(\boldsymbol{u}(k)):= & \sum_{i=1}^{N_{g}}\left[C_{i}^{\mathrm{DG}}\left(P_{i}(k)\right)+O M_{i} P_{i}(k)+S U_{i}(k)+S D_{i}(k)\right]+C^{g}(k) \\
& +O M^{b}\left|P^{b}(k)\right|+\rho_{c} \sum_{h=1}^{N_{c}} \beta_{h}(k) D_{h}^{c}(k) .
\end{aligned}
$$

We recall that $C^{g}(k)$ can be negative, i.e. energy is sold to the utility grid, representing a profit for the microgrid system. Depending on the DG, maintenance costs can be also expressed by the term $O M_{i} \delta_{i}(k)$.

Also $P^{b}(k)$ can be negative, i.e. discharging mode for the storage unit. Thus, it is necessary to include the absolute value, $\left|P^{b}(k)\right|$, in the objective function to account for costs associated with the use of the storage. The absolute value can be easily expressed as $\left(2 z^{b}(k)-P^{b}(k)\right)$.

Note that $V_{r}(\boldsymbol{u}(k))$ is a quadratic cost due to the presence of the quadratic terms $C_{i}^{\mathrm{DG}}\left(P_{i}(k)\right)$. We therefore approximate every function $C_{i}^{\mathrm{DG}}\left(P_{i}\right)$ with a convex piecewise affine function, which provides very similar results, but can be entered into a mixed integer linear program [53].

In particular, the fuel cost function of a DG, $C^{\mathrm{DG}}(P)=a_{1} P^{2}+a_{2} P+a_{3}$, is approximated by the max of affine functions without introducing binary variables as follows [65]

$C^{\mathrm{DG}}(P) \approx \max _{j=1, \ldots, n}\left\{G_{j} P+g_{j}\right\}=\|\boldsymbol{G} P+\mathbf{g}\|_{\infty}$,

where $P$ is the generated power and $G$ and $g$ are obtained by linearizing the function at $n$ points (the subscript $j$ extracts the $j$ th row of $G$ and $g$ ).

Microgrid emissions. The emission rate $(\mathrm{kg} / \mathrm{h})$ of atmospheric pollutants such as sulphur oxides $\mathrm{SO}_{2}$, carbon oxides $\mathrm{CO}_{2}$, and nitrogen oxides $\mathrm{NO}$ and $\mathrm{NO}_{2}$ can be modeled separately; the total emission of the $i$ th unit can be expressed as [66]:

$E_{i}(P)=v_{i}+\zeta_{i} P+\gamma_{i} P^{2}+\eta_{i} e^{\lambda_{i} P}$

where $v_{i}, \zeta_{i}, \gamma_{i}, \eta_{i}$ and $\lambda_{i}$ are nonnegative coefficients of the emission characteristics. We emphasize that, when a CHP unit is considered, the total emission of the unit can still be expressed as function of $P$ by (14), since ( 7$)$ is valid and $Q_{C H P}=(1 / P H R) P_{C H P}$. The microgrid emission at time step $k$ is denoted by $V_{e}(\boldsymbol{u}(k))$ and is given by

$V_{e}(\boldsymbol{u}(k)):=\sum_{i=1}^{N_{g}} E_{i}\left(P_{i}(k)\right)$.

Each function $E_{i}(P)$ can be approximated with a piecewise affine function in order to keep the problem linear [64].

We point out that we do not account explicitly for the pollutants content in the electricity purchased from the grid, which is hard to estimate and out of the scope of this study. However, the proposed optimization model can easily include emissions from utility grid (for instance, utility grid emissions can be modeled by a linear function of the purchased power whose coefficients represent time-varying emission rates).

\subsubsection{Capacity and terminal constraints}

To pose the final MILP optimization problem, additional operational constraints must be met [60]

$$
\begin{aligned}
& x_{\min }^{b} \leq x^{b}(k) \leq x_{\max }^{b} \\
& P_{i, \min } \delta_{i}(k) \leq P_{i}(k) \leq P_{i, \max } \delta_{i}(k)
\end{aligned}
$$


${ }_{431}\left|P_{i}(k)-P_{i}(k-1)\right| \leq R_{i, \max } \delta_{i}(k-1)$

$\beta_{h, \min } \leq \beta_{h}(k) \leq \beta_{h, \max }$

with $i=1, \ldots, N_{g}$ and $h=1, \ldots, N_{c}$. The constraints above model the physical bounds on the storage device (inequality (15a)), the power flow limits of the DG units (inequality (15b)) and their ramp up and ramp down rates (inequality (15c)), the bounds on flexible loads curtailments (inequality (15d)).

\subsubsection{A stochastic programming approach with simple recourse}

In the microgrid scenario, recourse actions are needed when the actual energy demand is different from the actual energy generation in the microgrid and system performance naturally degrades: higher demand than generation leads to energy shortage, while lower demand than generation results in unexpected energy surplus. In case of power imbalances, technically the utility grid can cover it and charge the microgrid operator according to special agreement between the microgrid operator and the Distribution System Operator (DSO). Power imbalances are represented through recourse variables in the stochastic optimization problem. For instance, in case of energy deficit during a certain time, a recourse action can be taken, i.e. the needed amount of energy is purchased from the utility grid at real-time energy prices: the corresponding recourse variable represents the needed amount of energy to be purchased in order to keep the power balance at that point in time.

The power balance constraint (2.4) at each time $k, \boldsymbol{F}(k) \boldsymbol{u}(k)+$ $\boldsymbol{f} \boldsymbol{w}(k)=0$ is the random constraint, or second-stage constraints, in the optimization model described in Section 3.1; all the other constraints can be considered as first-stage constraints.

Given an initial time step $\kappa$ and a time duration $T$, denote the initial storage level at time $\kappa$ by $x^{b}(\kappa)$ and define

$\xi_{\kappa}^{+, T}:=\left[\xi^{+}(\kappa) \ldots \xi^{+}(\kappa+T-1) \quad \xi_{q}^{+}(\kappa) \ldots \xi_{q}^{+}(\kappa+T-1)\right]^{\prime}$,

$\xi_{\kappa}^{-, T}:=\left[\xi^{-}(\kappa) \ldots \xi^{-}(\kappa+T-1) \quad \xi_{q}^{-}(\kappa) \ldots \xi_{q}^{-}(\kappa+T-1)\right]^{\prime}$,

$\boldsymbol{u}_{\kappa}^{T}:=\left[\boldsymbol{u}^{\prime}(\kappa) \ldots \boldsymbol{u}^{\prime}(\kappa+T-1)\right]^{\prime}$,

$\boldsymbol{w}_{\kappa}^{T}:=\left[\boldsymbol{w}^{\prime}(\kappa) \ldots \boldsymbol{w}^{\prime}(\kappa+T-1)\right]^{\prime}$.

First-stage function. We remind that, in the two-stage stochastic framework, the first-stage cost function and constraints do not depend on random variables. The first-stage constraints are: (i) storage model (5); (ii) constraints (2), (9) and (10); (iii) capacity and terminal constraints (15).

Since we account for both economical and environmental costs, to compute the first-stage plan of microgrid operations we apply the weighted min-max method.

Define $\hat{V}_{i}\left(\boldsymbol{u}_{\kappa}^{T}\right)=\sum_{k=0}^{T-1} V_{i}(\boldsymbol{u}(\kappa+k)), i \in\{r, e\}$.

A normalization of objectives is required to get a Pareto optimal solution consistent with the assigned weights since different objective functions can have different magnitude. In this study we adopt one of the most effective normalization scheme, based on the utopia and nadir points [55]. We call $V_{i}^{o}$, with $i \in\{r, e\}$, the utopia point, i.e. the ideal value obtained by minimizing each of the objective functions individually subject to the first-stage constraints. The nadir point is $\hat{V}_{i}^{\max }=\max _{j \in\{r, e\}} \hat{V}_{i}\left(\boldsymbol{u}_{j, \kappa}^{T}\right)=\hat{V}_{i}\left(\boldsymbol{u}_{j \neq i, \kappa}^{T}\right)$, where $\boldsymbol{u}_{j, \kappa}^{T}$ is the point that minimizes the $j$ th objective function and $i \in\{r$, $e\}$. The utopia and the nadir points provide respectively a lower and an upper bound of the Pareto optimal set.

Consider then the following cost function

$J_{f s}\left(x^{b}(\kappa), \boldsymbol{u}_{\kappa}^{T}\right)=\max _{i \in\{r, e\}}\left\{\theta_{i} \tilde{V}_{i}\left(\boldsymbol{u}_{\kappa}^{T}\right)\right\}$ where $\tilde{V}_{i}\left(\boldsymbol{u}_{\kappa}^{T}\right)$ is the normalized objective function $i$

$\tilde{V}_{i}\left(\boldsymbol{u}_{\kappa}^{T}\right)=\frac{\hat{V}_{i}\left(\boldsymbol{u}_{\kappa}^{T}\right)-V_{i}^{o}}{\hat{V}_{i}^{\max }-V_{i}^{o}}$,

and the nonnegative weights $\theta_{i}$ are such that $\sum_{i \in\{r, e\}} \theta_{i}=1$.

All the objective functions after normalization will take values between zero and one, depending on the accuracy with which the utopia and the nadir points are computed.

Since in an MPC scheme can be computationally expensive and challenging to obtain the utopia and the nadir points at each point in time, approximations can be used (e.g., considering the bestcase scenario of load and PV generation profiles over the prediction horizon for the utopia point, based on historical data) [55].

Second-stage function. At the second stage we aim at minimizing the expected value of the costs due to power imbalances. In the case of discrete distribution of random variables with a finite number $S$ of scenarios, $\hat{\boldsymbol{w}}_{1, \kappa}^{T}, \ldots, \hat{\boldsymbol{w}}_{S, \kappa}^{T}$, with corresponding probability $p_{1}, \ldots$, $p_{S}$, the second-stage function can be written as

$$
\begin{aligned}
J_{s S}\left(\boldsymbol{u}_{\kappa}^{T}, \boldsymbol{w}_{\kappa}^{T}\right)= & \min \sum_{i=1}^{S} p_{i}\left(\boldsymbol{q}^{+} \boldsymbol{\xi}_{i, \kappa}^{+, T}+\boldsymbol{q}^{-} \boldsymbol{\xi}_{i, \kappa}^{-, T}\right) \\
\text { s.t. } & \\
& \boldsymbol{\xi}_{i, \kappa}^{+, T} \geq \tilde{\boldsymbol{F}}_{\kappa} \boldsymbol{u}_{\kappa}^{T}+\tilde{\boldsymbol{f}} \hat{\boldsymbol{w}}_{i, \kappa}^{T} \\
& \boldsymbol{\xi}_{i, \kappa}^{-, T} \geq-\left(\tilde{\boldsymbol{F}}_{\kappa} \boldsymbol{u}_{\kappa}^{T}+\tilde{\boldsymbol{f}} \hat{\boldsymbol{w}}_{i, \kappa}^{T}\right) \\
& \boldsymbol{\xi}_{i, \kappa}^{+, T}, \boldsymbol{\xi}_{i, \kappa}^{-, T} \in \mathbb{R}_{+} \\
& \forall i=1, \ldots, S
\end{aligned}
$$

where $\boldsymbol{q}^{+}$and $\boldsymbol{q}^{-}$are costs related to energy surplus and shortage respectively, $\tilde{\boldsymbol{F}}_{\kappa}=\operatorname{diag}(\boldsymbol{F}(\kappa), \ldots, \boldsymbol{F}(\kappa+T-1))$ and $\tilde{\boldsymbol{f}}=\boldsymbol{f} \otimes I_{T \times T}$, with $\otimes$ denoting the Kronecker product.

Problem formulation. The two-stage stochastic problem for microgrid economic/environmental operation management can be formulated as follows

s.t.

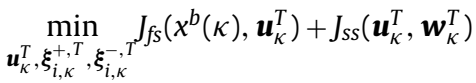

first-stage constraints.

We apply the weighted min-max method [55], hence the optimization problem above can be reformulated as the following mixed integer linear program

s.t.

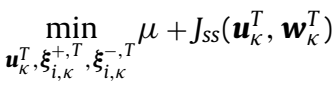

$$
\theta_{i} \tilde{V}_{i}\left(\boldsymbol{u}_{\kappa}^{T}\right) \leq \mu \quad \forall i \in\{r, e\}
$$

first-stage constraints,

where $\mu$ is a scalar auxiliary variable introduced to model the max in (16). Compared to the microgrid operation optimization problem in [60], Problem (18) introduces 2NT additional continuous variables and $4 N T$ additional constraints.

We remark that the stochastic problem we propose has complete recourse.

\subsection{Uncertainty modeling and scenario generation}

In order to make the proposed control action effective, the stochastic nature of RES and demand is incorporated in the MPC 
problem through scenarios. Denote by $\omega_{k}$ the uncertainty during the sampling period $k$, which can represent either the energy demand or the RES generation. The random variable $\omega_{k}$ is decomposed as $\omega_{k}=\bar{\omega}_{k}+\tilde{\omega}_{k}$ where $\bar{\omega}_{k}$ is the forecast and $\tilde{\omega}_{k}$ is the forecast error at time $k$. In this study, a Least-Squares Support Vector Regressor [67-69] is applied for forecasts $\bar{\omega}_{t}$ for the whole planning horizon $t=1, \ldots, T$.

The main issue for finding a solution of a stochastic program is the necessity to model uncertainty such that the corresponding optimization problem is numerically tractable. An optimization problem that incorporates uncertainties represented by multidimensional continuous distributions or by discrete distributions with large number of outcomes cannot be easily handled. To overcome this difficulty, one possible solution is to approximate the actual probability distribution of the uncertainty as a discrete distribution with a tractable number of representative outcomes, which occur with a certain probability. This means that a tradeoff is needed between the accuracy of uncertainty model and the tractability of the corresponding optimization problem. To build the discrete distribution for the uncertainty, it is quite natural to use historical data, if available. In this work, historical data are employed to train a hidden Markov model (HMM) for generating the finite number of paths, i.e. scenarios, and the corresponding probability of occurrence $[70,71]$; the resulting set of paths is called fan. To properly decrease the scenario number and make the stochastic problem tractable, scenario reduction algorithms can be applied. The Backward Reduction Algorithm has been proved to provide very good performances in the two-stage mixed integer stochastic programming framework [72,21].

The accuracy of the scenario generation approach will affect the quality of decision making. We will not argue about the statistical property of the computed approximation, but we evaluate its effectiveness through the improvement in the solution quality. However, we emphasize that the focus of this paper is not the scenario generation mechanism but the optimization-based control framework for microgrid management systems; a different scenario generation mechanism can be easily integrated in the proposed framework.

\subsection{Stochastic MPC formulation}

The scenarios generated by employing the mechanism outlined above is incorporated in the two-stage stochastic formulation (18) in order to make the solution more robust, i.e. less sensitive to changes in the energy demand and RES generation. The microgrid operational plan can be further improved by introducing a feedback mechanism, i.e. applying a receding horizon philosophy.

The stochastic microgrid controller is implemented online according to Algorithm 1.

\section{Algorithm 1. Online implementation}

1: $\quad$ for $k=1,2, \ldots$ do

2: $\quad$ measure $x^{b}(k)$

3: $\quad$ compute the scenarios $\hat{\boldsymbol{w}}_{i, k}^{T}, i=1, \ldots, S$

4: $\quad$ compute $\boldsymbol{u}_{k}^{T}$ by solving Problem (18)

5: $\quad$ apply the first sample of the input sequence, $\boldsymbol{u}_{k}$

6: $\quad$ shift the prediction horizon

\section{Extended modeling}

In this section we extend the microgrid optimization problem formulation presented in Section 3.2. The first-stage constraints can be extended in order to model the reactive power management and solve a combination of active and reactive power management problem for microgrid. In order to guarantee the power quality and the grid stability, once the optimal active power setpoints have been computed the reactive power has to be adjusted for all the microgrid components; generating resources, which operate within their capability limits, are required by ISOs to produce or absorb reactive power to maintain voltages within limits. In order to define suitable setpoints of the reactive power, either the local droop controllers can be used, which is the case considered in this work, or optimal values can be computed through optimization.

Let us denote by $R_{p}^{\mathrm{dg}}, R_{p}^{\text {load }}$ and $R_{p}^{\text {grid }}$ the total reactive power respectively from distributed generators, loads and the utility grid. To include the reactive power management in Problem (18), we need to add the following constraints:

- the reactive power balance

$$
R_{p}^{\mathrm{dg}}+R_{p}^{\text {load }}+R_{p}^{\text {grid }}=0
$$

- the relationship between reactive and active power for each microgrid component $i$, given by

$$
\left(P^{i}\right)^{2}+\left(R_{p}^{i}\right)^{2} \leq\left(S^{i}\right)^{2}
$$

where $S^{i}$ is a known parameter (i.e. the nominal apparent power) given by the capability curve of the component (see [73] and references within).

There could be two possible scenarios while considering the reactive power exchanged with the utility grid $R_{p}^{\text {grid }}$ : (i) the microgrid is required by ISOs to supply a total amount of reactive power to the grid, which entails $R_{p}^{\text {grid }}$ being a given parameters to the microgrid optimization problem; (ii) $R_{p}^{\text {grid }}$ is an additional decision variable, which is computed so that the voltage is improved at local points of the microgrid.

This formulation provides additional flexibility to the problem since it allows to treat the reactive power as ancillary services in a deregulated electricity markets, where financial compensation schemes are created for reactive power providers.

By including the constraints outlined above in Problem (18), the optimization problem will become a mixed integer quadratically constrained linear problem, which can still be solved by standard algorithms and solvers, e.g. CPLEX.

We eventually remark that, in case the microgrid is isolated, the problem formulation can be easily adapted by removing the interaction with the utility grid; however, backup storage capabilities have to be considered.

\section{Experimental results}

In this section we will discuss experimental results.

\subsection{Description of the experimental setup}

The experimental evaluation of the control algorithm was performed at the Center for Renewable Energy Sources and Saving (CRES), Pikermi - Athens, Greece. The microgrid runs on a low-voltage 3-phase electric network with all DER components connected, an Interbus communication and control network, a Modbus based monitoring of active and reactive power at the mains, a graphical interface for supervision, monitoring and control. The experimental microgrid of CRES comprises the following units (see Fig. 1):

- two photovoltaic generators 1.1 and $4.4 \mathrm{kWp}$ (the second on a single-axis tracking system);

- two battery storage systems with total capacity $80 \mathrm{kWh}$, each consisting of 2V FLA (Flooded Lead Acid) battery cells and interconnected to the mains through battery inverters which offer numerous control capabilities, such as grid-forming, grid-tied and droop-mode; it is worth mentioning that during the tests one 


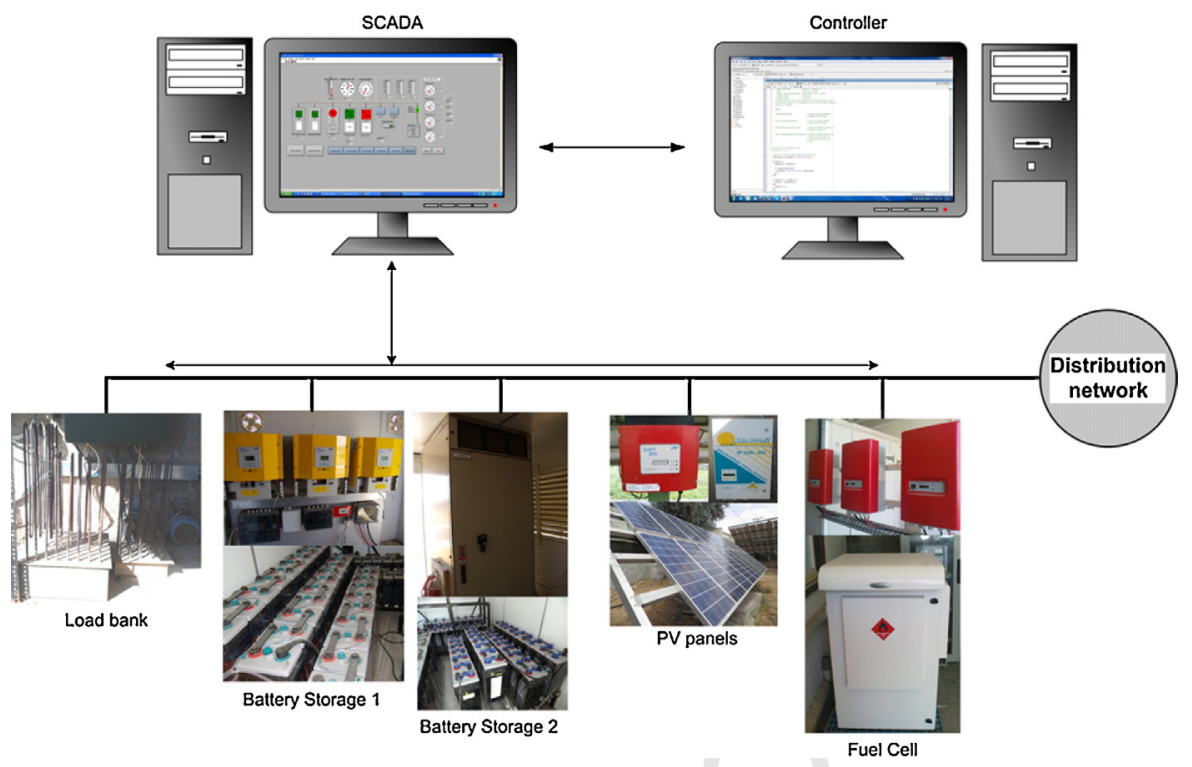

Fig. 1. Experimental microgrid setup.

of the two battery systems was used to simulate the operation of a micro-CHP.

- a load bank of resistors of maximum active power $13 \mathrm{~kW}$. This load is equally distributed into the three phases. The loads are grouped into Flexible and Critical loads and programmed by using data files of consumption so as to produce the desired profiles;

- one $5 \mathrm{~kW}$ Proton Exchange Membrane (PEM) fuel cell equipped with a DC/AC 3-phase system. Hydrogen consumption is $40 \mathrm{NL} / \mathrm{min}$ at $3 \mathrm{~kW}$ and $75 \mathrm{NL} / \mathrm{min}$ at $5 \mathrm{~kW}$. The fuel cell is supplied with a compressed hydrogen storage tank at a maximum pressure of 16 bar, a physical volume of $3000 \mathrm{~L}$ and a nominal hydrogen storage capacity of $50 \mathrm{~N} \mathrm{~m}^{3}$;

- one PEM electrolyser producing hydrogen at $0.5 \mathrm{~N} \mathrm{~m}^{3} / \mathrm{h}$ at a pressure of up to 14 bar.

Low level controllers for microgrid components were available:

- a local PI controller was employed to regulate the battery inverter frequency according to the active power set point;

- a local PI controller was used for the $\mu \mathrm{CHP}$ inverter;

- the fuel cell unit was regulated by using a local controller and a comparator with the mean power reference value;

- concerning the load bank, a local controller selected the most appropriate combination of resistors which provided the closest consumption value according to the set-point.

The control algorithm was implemented on a PC interfaced with the microgrid SCADA system through the Local Area Network. The results of four experiments (run over a 2-month period) are presented:

- Experiment MG-DMPC ${ }_{1}$ : the microgrid is operated by the deterministic MPC control scheme on Day 1 . The controller is set with total weight on operation costs;

- Experiment MG-SMPC 1 : the microgrid operations are managed by the MG-SMPC control scheme on Day 2 . The controller is set with total weight on operation costs;

- Experiment MG-SMPC 2 : the microgrid operations are managed by the MG-SMPC control scheme on Day 3. The controller is set

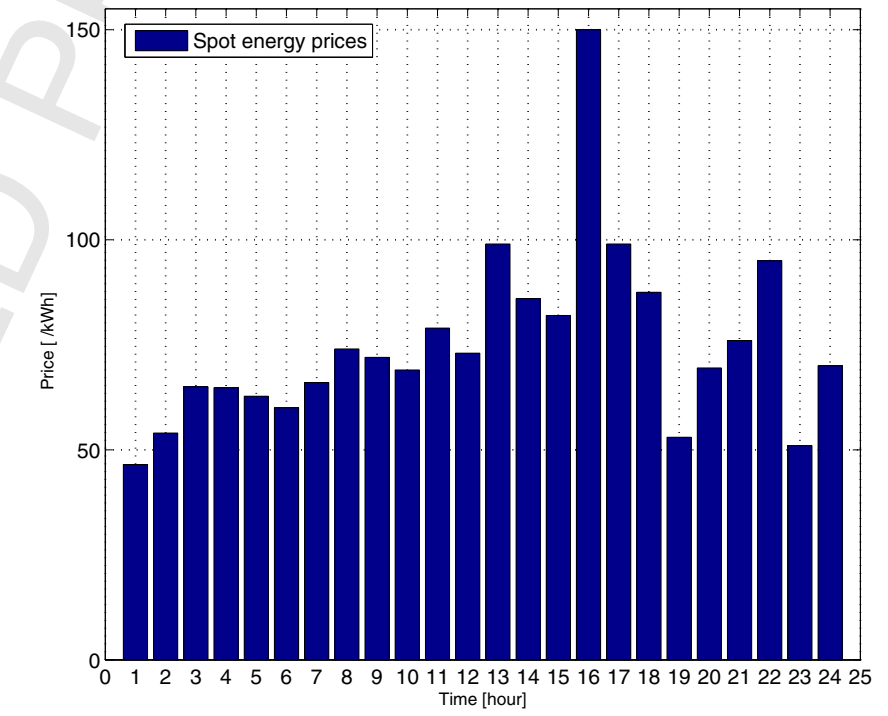

Fig. 2. Spot energy prices over $24 \mathrm{~h}$ (all experiments).

with $\theta_{r}=0.7$ (weight on operating costs) and $\theta_{e}=0.3$ (weight on emissions);

- Experiment MG-SMPC 3 : the microgrid operations are managed by the MG-SMPC control scheme on Day 4 . The controller is set with $\theta_{r}=0.3$ (weight on operating costs) and $\theta_{e}=0.7$ (weight on emissions).

The daily spot prices are from the EEX and are shown in Fig. 2. All data used in experiments are realistic; they are based on measurements, datasheets and market prices. Loads follow a realistic profile derived from real consumption measurements from CRES; in particular, thermal loads determine the energy demand profile of a heat pump employed in the research center. We consider both flexible and critical electrical loads. The electrical and thermal loads profiles are shown in Figs. 3 and 4. During all the four experiments, the system is operated with the same consumption profiles. 


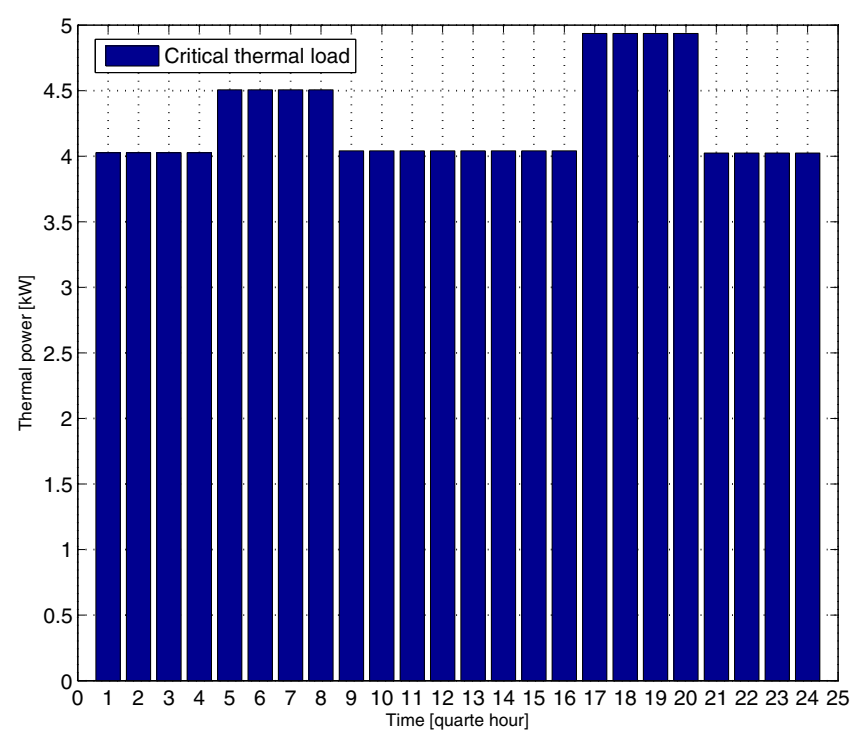

Fig. 3. Thermal load over 24 time steps ( $6 \mathrm{~h}$ ) (all experiments).

The MPC-based controllers are run with a planning horizon of 48 time steps. We consider a 15-min sampling period and the experiments are run over $6 \mathrm{~h}$. The experiments started after dawn so the PV units generate some power at all time steps (see Fig. 5 for an example of PV power generation during one of the experiment days).

We apply Least-Square Support Vector Machines (LS-SVM) for regression (LS-SVR, Support Vector Regression) with a moving time window to forecast the renewable power generation for the hours ahead based on historical data. Forecast errors based on historical data are also employed to train a HMM, as illustrated in Section 3.3. At each time step (15 $\mathrm{min}$ ) the regressor and the HMM are updated with the most recent available measurement, while the oldest data are discarded.

In order to evaluate the suitability of the scenario generation method for the proposed stochastic programming model, we performed the stability tests as described in [21]. As a benchmark scenario fan, we consider historical data in the period from September to October 2012 as a representation of the true distribution.

Then, for a given size of the scenario fan, we generated 25 scenario fans, solved the corresponding scenario-based optimization problems, and investigated whether the solutions were independent from the particular scenario fan and how far the solutions obtained are close to the "true" solution derived from the benchmark fan. We found that a fan of 80 scenarios can guarantee good performance.

See $[60,61]$ for an outline of the forecasting algorithm and parameters estimation, such as investment and operating costs, minimum up and down times of the DG units, efficiencies, storage limits.

In the following paragraph we give some details on thermal generation and demand in the experimental setup.

Thermal generation and demand. Due to the fact that the implemented control strategy aims at optimizing the system operation in terms of thermal needs coverage and $\mathrm{CO}_{2}$ emissions reductions, it is important to achieve an estimation of two critical quantities, i.e. the Power/Heat Ratio (PHR) of the selected CHPs and the $\mathrm{CO}_{2}$ emissions which correspond to the required operating point. The thermal load profile to be covered by the CHPs during the experiment is derived from measurements in one of the CRES buildings. Specifically, these measurements are related to a heat pump whose COP (Coefficient of Performance) is known. By measuring its

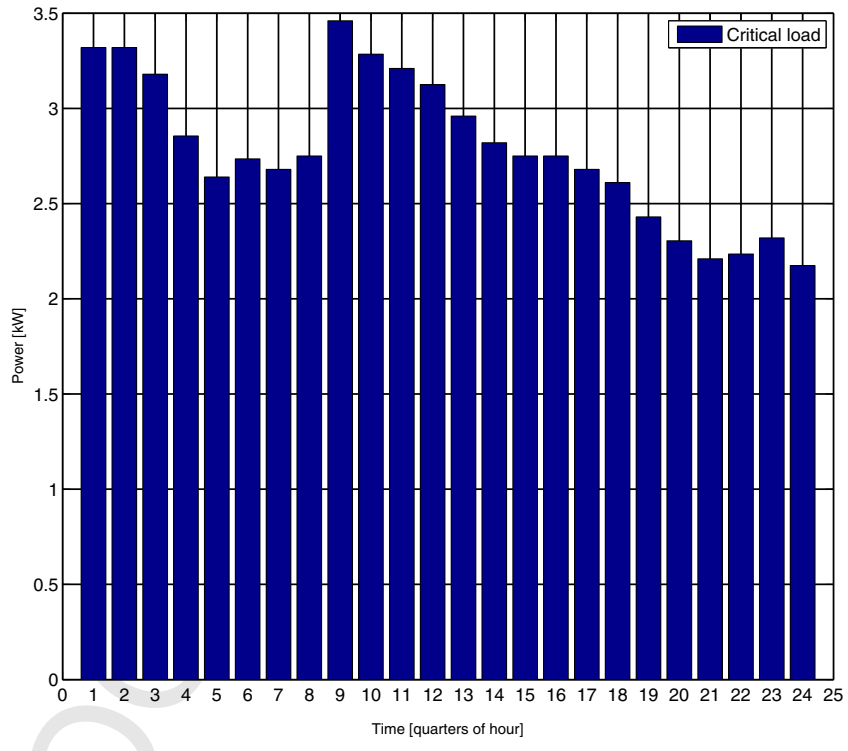

(a) Critical load

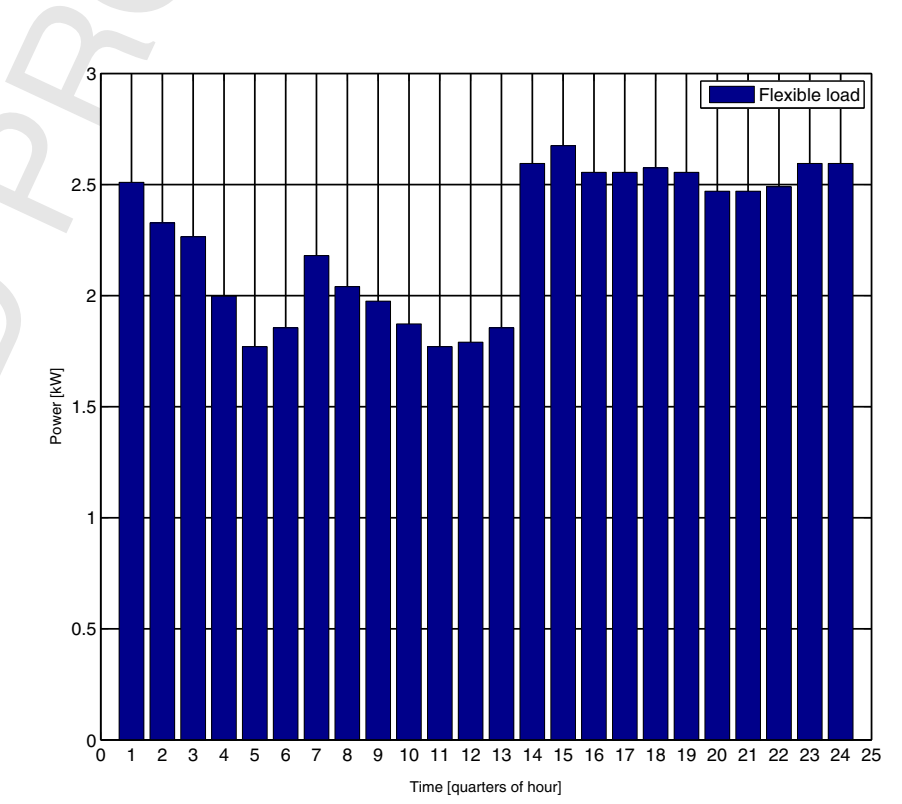

(b) flexible load

Fig. 4. Critical and flexible electrical loads over 24 time steps ( $6 \mathrm{~h}$ ) (all experiments).

electricity consumption during one winter day and multiplying it by the COP ratio, one gets the thermal production of the heat pump averaged in 15-min, as shown in Fig. 6. Then such total amount of thermal need is scaled down to the size of our study and then distributed to the two units according to their power levels and PHRs.

The knowledge or estimation of PHR for the two CHP units is necessary in order to estimate the electricity produced for the requested thermal load and vice versa. The optimization algorithm is then responsible to trade-off thermal needs coverage with the optimum electrical operation of the system. In this study PHR is estimated for the power level of the selected units by tables from literature [74] which show empirical values of efficiencies and PHRs for different CHP technologies.

The PHR for both considered CHPs was estimated as the lowest values of the right column of Table 4 . This was due to the selected power level of the units. Thus, we considered for the fuel cell unit 


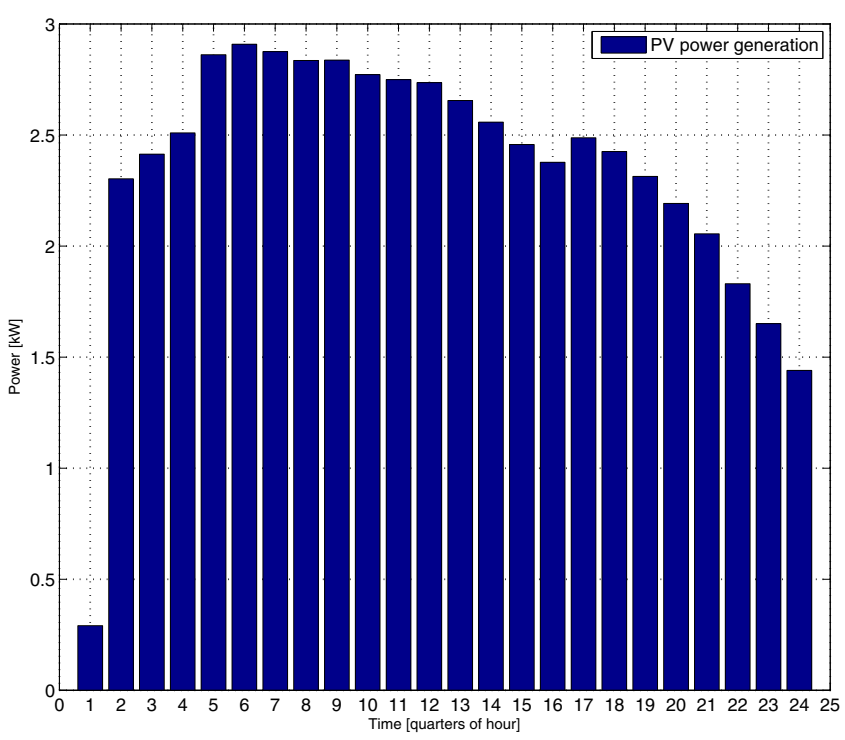

Fig. 5. PV generation profile over 24 time steps (6h) on Day 3.

Table 4

Power/heat ratio, electrical and thermal efficiencies for the two types of CHPs considered.

\begin{tabular}{lclll}
\hline & Electrical power & $\eta_{e}(100 \%$ load $)$ & $\eta$ & PHR \\
\hline Fuel cell & $0.04-50 \mathrm{MW}$ & $37-45$ & $85-90 \%$ & $0.8-1.0$ \\
CHP & $0.015-2 \mathrm{MW}$ & $27-35$ & $60-80 \%$ & $0.5-0.7$ \\
\hline
\end{tabular}

a PHR equal to 0.8 while for the reciprocating engine the PHR was assumed 0.5 .

Concerning the $\mathrm{CO}_{2}$ emissions of the two CHPs, the total amount of $\mathrm{CO}_{2}$ is calculated knowing the consumed fuel according to $m_{\mathrm{CO}_{2}}=3.6667 c\left(E / \eta H_{u}\right)$, where $c$ is the mass content of carbon in fuel (e.g. ( $\mathrm{kg}$ of $\mathrm{C}) /(\mathrm{kg}$ of fuel $)), E$ is the useful energy produced by the system, $\eta$ is efficiency of the system, based on the lower heating value of fuel and $H_{u}$ lower calorific value of the consumed fuel. For natural gas the mass content is $75 \%$ while $H_{u}$ is equal to $49,000 \mathrm{~kJ} / \mathrm{kg}$. The amount of energy is the total energy (electrical plus thermal) produced by the unit in a time frame of $15 \mathrm{~min}$.

Experimental results evaluation. Table 5 reports the experimental results in terms of operating costs and emissions. Costs reported taken to meet the power balance at each time step, i.e., costs to compensate for actual power imbalances. In case of power deficit or surplus, several options are available: the deficit of power can be purchased from the utility grid or discharged from the battery, in Table 5 include the costs of corrective actions that have to be
Table 5

Results evaluation

\begin{tabular}{lcll}
\hline & PV yield $(\mathrm{kWh})$ & Costs $(€ / \mathrm{kWh})$ & Emissions $(\mathrm{kg} / \mathrm{kWh})$ \\
\hline MG-DMPC $_{1}$ & 3.43 & 0.2705 & 0.1083 \\
MG-SMPC $_{1}$ & 9.93 & 0.1747 & 0.0778 \\
MG-SMPC $_{2}$ & 13.76 & 0.2204 & 0.0777 \\
MG-SMPC $_{3}$ & 14.37 & 0.47 & 0.0438 \\
\hline
\end{tabular}

or controllable loads can be further curtailed, while the excess of power can be either sold to the grid, or used to charged battery, or possible curtailments can be reduced. The feasibility of the aforementioned options must be verified, since constraints on curtailments and power exchange with battery and grid must be satisfied. Nearly all the imbalances during the experiments are due to power deficit, which is then purchased from the grid. However, we associate a cost to any of the options described above, in order to account for possible costs and penalties due to real-time imbalances, as well as for the non-optimality of the resulting power dispatch.

In order to assess performance of the proposed control framework independently of disturbance profiles (i.e. PV power output in this case study), costs reported in Table 5 are average costs per kWh of supplied energy, which includes energy sold to the grid but not the energy generated by the photovoltaic plant.

We can see that the stochastic strategy focusing on operating costs, MG-SMPC 1 , yields a $30.5 \%$ savings over the deterministic one, MG-DMPC .

During all experiments with stochastic controllers, imbalances occur mainly in the morning, at the beginning of the experiments, when less data are available to train the regressor and the HMM. During the experiments with MG-SMPCs imbalances are reduced by $54 \%$ compared to the experiments with the MG-DMPC.

The cost of power shortage or surplus in the stochastic controllers is set to 10 , which is at least one order of magnitude higher than the other costs. Curtailments are usually penalized since they lead to user discomfort; so they are not performed unless strictly convenient or necessary. We set a not so high weight on curtailment, $\rho_{c}=0.1$ : this way the optimization algorithm uses curtailments when more economically convenient.

In the following we discuss more into detail the experiments run on Days 3 and 4, showing the differences in microgrid operations resulting from a different weighting in the cost function.

Curtailments occur when there are power peaks in flexible loads and the prices are higher (see Fig. 7).

The battery is employed mainly to gain some profit by selling power to the utility grid. The battery is always discharged at the same time steps when curtailments are required; this allows to sell

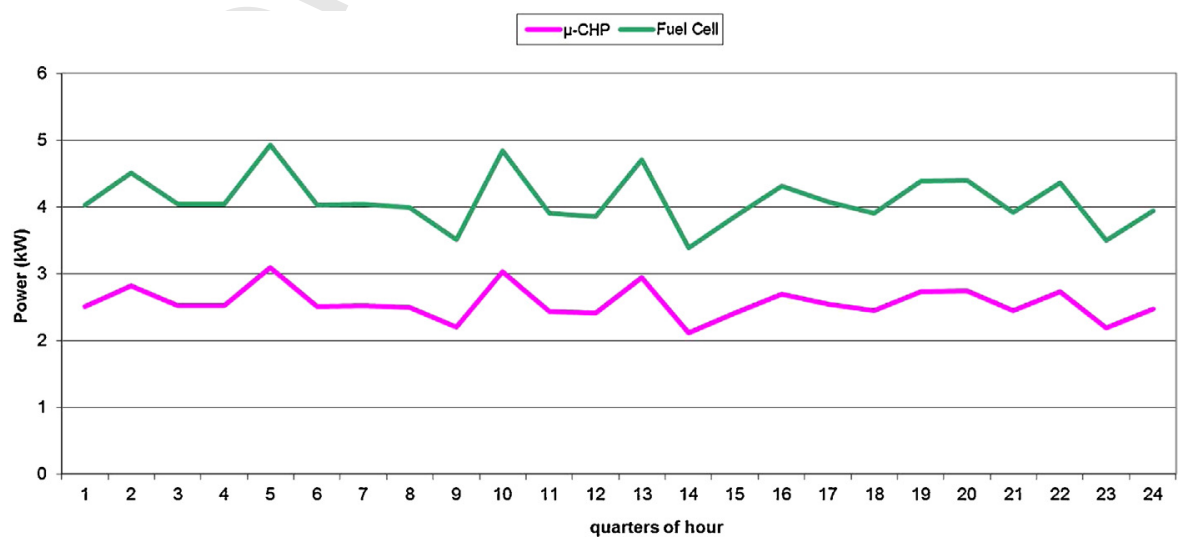

Fig. 6. Thermal production profile of the two CHPs. 


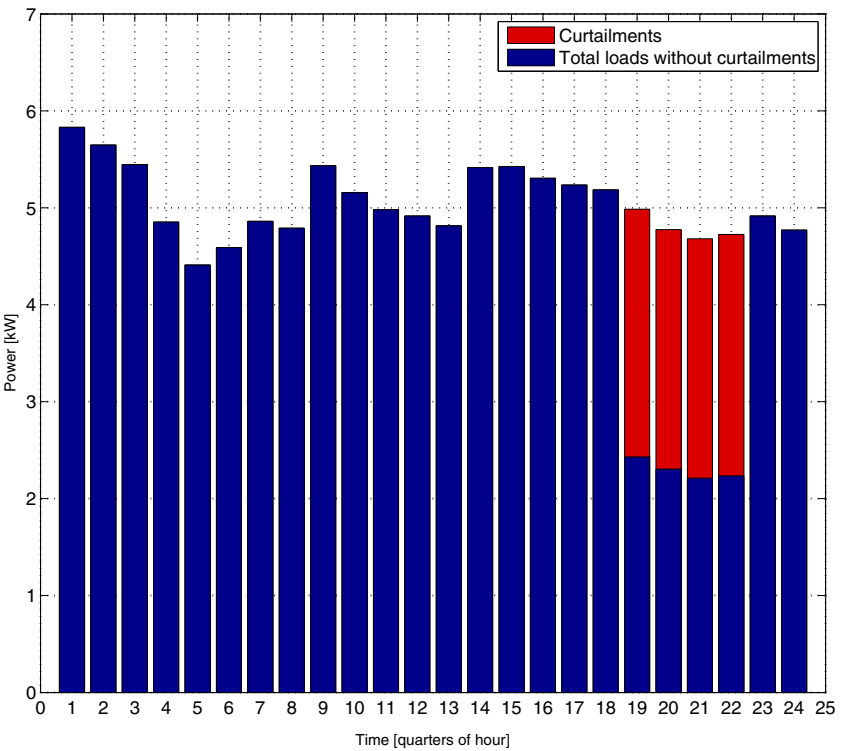

(a) MG-SMPC on Day 3

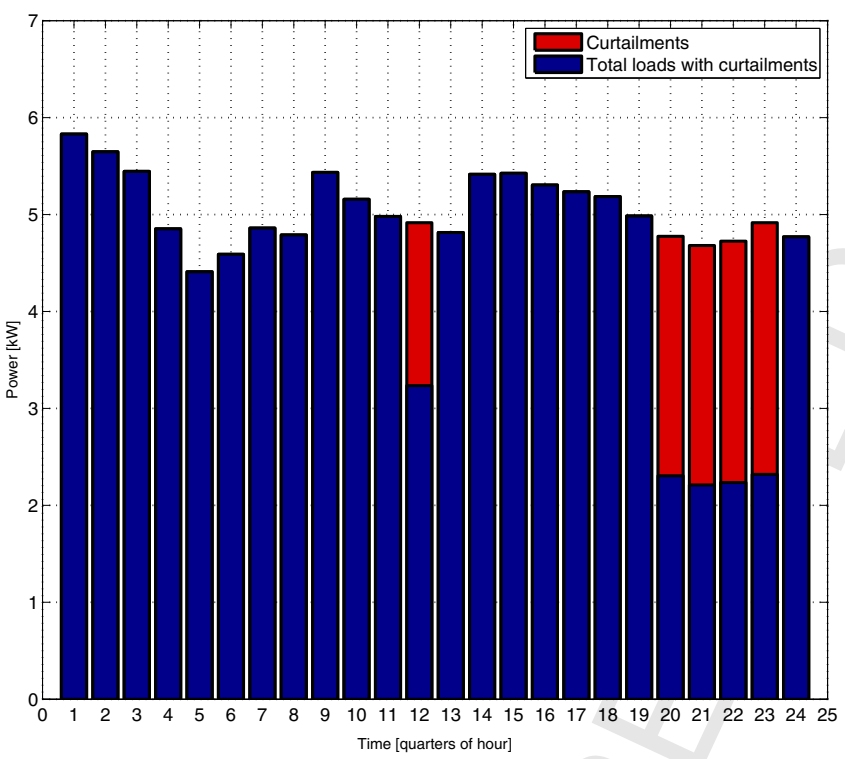

(b) MG-SMPC on Day 4

Fig. 7. Curtailments over 24 time steps (6h).

power to the utility grid while the PV plant and the distributed generators are employed to support the loads.

The fuel cell has generally large operative and maintenance costs and a low efficiency in electrical power generation, but it generates a rather smaller amount of emissions and can be employed to satisfy thermal loads. The fuel cell is then heavily used on Day 4, when the preferred objective is to reduce emissions, while it is much less utilized on Day 3, when the preferred objective is to reduce costs, due to its high maintenance costs (see Fig. 9). Since the weight on operating costs is greater than zero, the controller aims at compensating the higher costs due to the use of the fuel cell by increasing the curtailments and use the local generated power to sell power. Hence, on Day 4, some power is sold during the price peak at $11 \mathrm{am}$ which requires to curtail the flexible load during the price peak at $1 \mathrm{pm}$; this happens because the ultimate purpose of this experiment is to minimize the emissions, but with an eye on the costs (see Fig. 8).

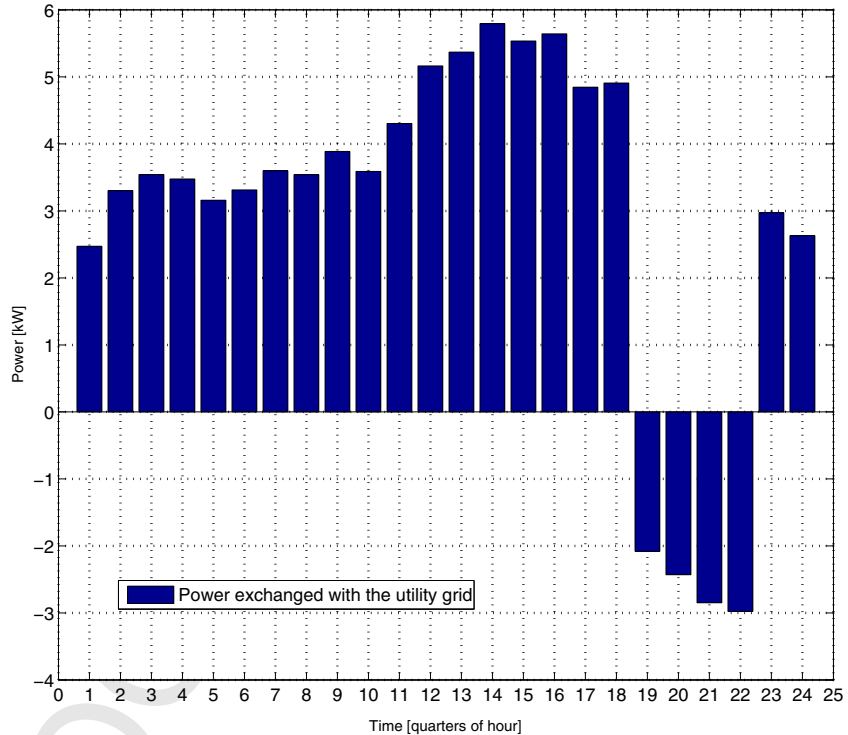

(a) MG-SMPC on Day 3

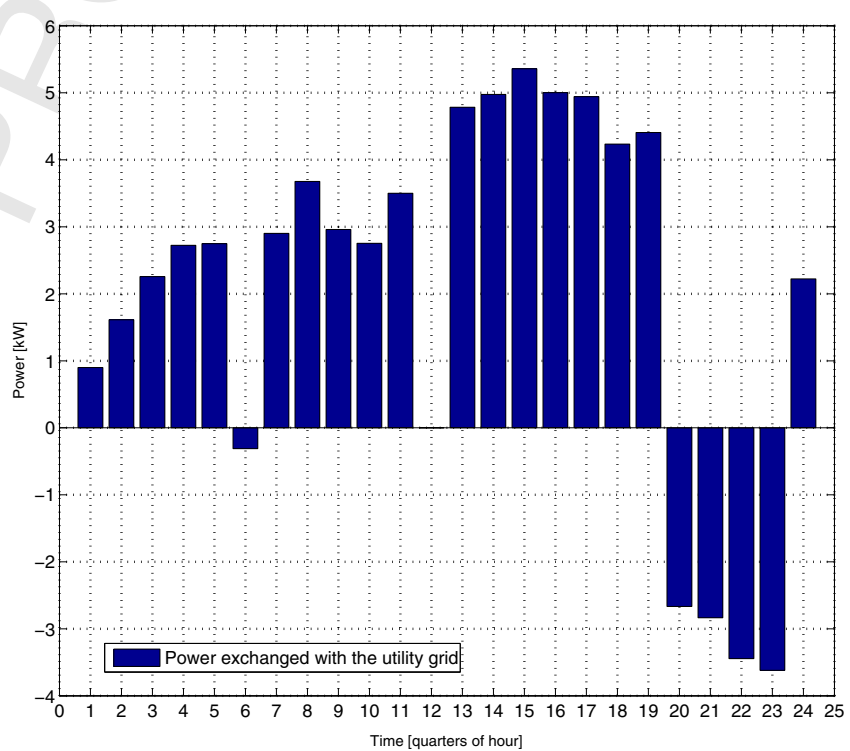

(b) MG-SMPC on Day 4

Fig. 8. Power exchanged with the utility grid over 24 time steps (6h).

Computational time for each iteration is less than $1.5 \mathrm{~s}$ on average.

Implementation and computational details. It is well known that MILP problems are NP-complete and their computational complexity mainly depends on the number of integer variables [56,53]. We point out that only continuous, and not binary, variables are added in the stochastic formulation. The formulation presented in the previous section was implemented using MatLab. We used ILOG'S CPLEX 12.0 [75] to solve the MILP optimizations, which is an efficient solver based on the branch-and-bound algorithm. All computations are done on an Intel Core 2 Duo CPU, $2 \mathrm{GHz}$. All the forecasts are obtained by Matlab's SVM (Support Vector Machine) toolbox, a LS-SVM training and simulation environment written in C-code [76]. The Hidden Markov Models are computed by using the Hidden Markov Model Toolbox for Matlab [77]. 


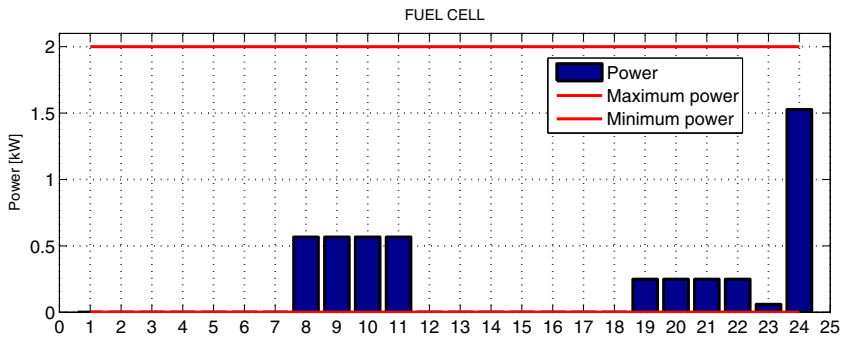

CHP

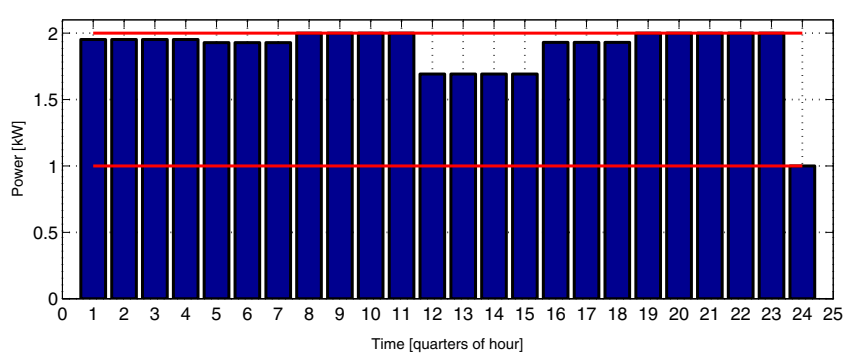

(a) MG-SMPC on Day 3
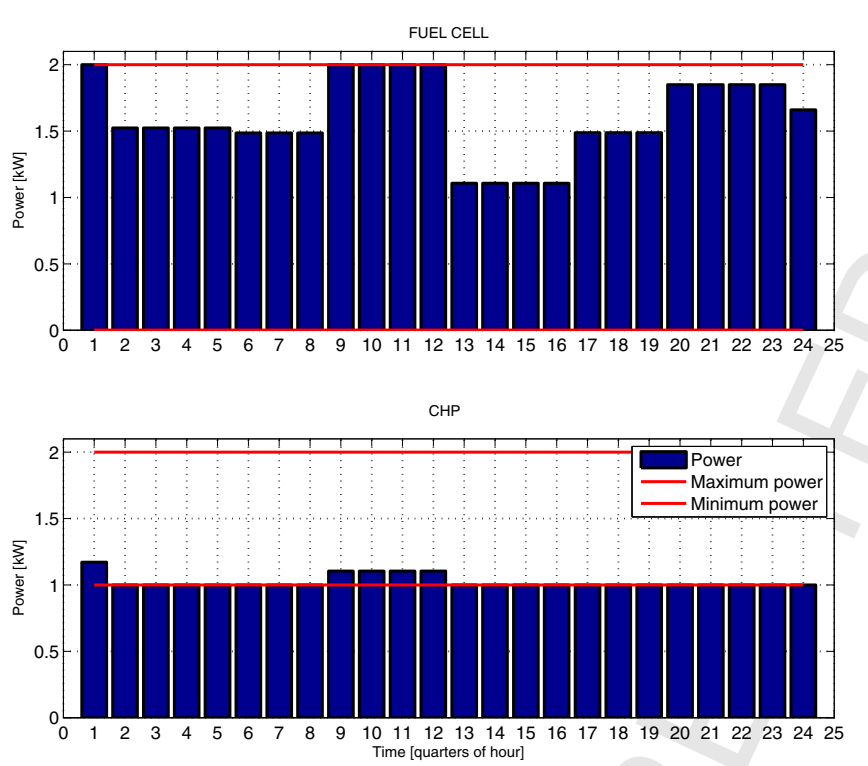

(b) MG-SMPC on Day 4

Fig. 9. CHP and fuel cell power generation over 24 time steps (6h).

\section{Conclusions and future studies}

In this paper, we present a control-oriented stochastic approach to microgrid modeling and high level economic/environmental optimization. Our main contributions are: (i) the development of an integrated optimization-based framework for microgrid operational planning which takes into account both thermal demand and emissions, as well as the inherent uncertainty due to renewable generation and energy demand; (ii) the experimental evaluation of the proposed control framework on an experimental microgrid. Modeling extensions to the reactive power management are also illustrated.

Experimental results show that the proposed control framework is feasible and implementable and that the stochastic controller can outperform the deterministic one. Further it is possible to use differently the available controllable energy source, as well as the flexible loads, by setting different weights on the two objectives (microgrid operating costs and emissions).

Currently stability and feasibility properties are under studies though extensive numerical computations have given reassuring results. The stability and feasibility of MPC are also a key concern. In the specific context, stability and constraints satisfaction should be guaranteed under uncertainty; intuitively speaking, it should be ensured that enough energy is stored in storage elements to counteract an unpredicted change in demand and RES generation. This can be ensured by a terminal set for the storage level which is a robust control invariant set, or by constraints tightening $[78,79]$.

Future work will also focus on analyzing the scalability of the proposed control framework and investigating distributed approaches.

\section{Acknowledgement}

The authors are grateful to the Department of PVs and DER systems, Center for Renewable Energy Sources and Saving (CRES) for the support provided during the experiments.

\section{Appendix A. Matrices}

$$
\begin{aligned}
& \mathrm{E}_{1}{ }^{b}:=\left[\begin{array}{llllll}
C^{b} & -\left(C^{b}+\varepsilon\right) & C^{b} & C^{b} & -C^{b} & -C^{b}
\end{array}\right]^{\prime} \\
& \mathrm{E}_{2}{ }^{b}:=\mathrm{E}_{2}{ }^{g}=\left[\begin{array}{llllll}
0 & 0 & 1 & -1 & 1 & -1
\end{array}\right]^{\prime} \\
& \mathrm{E}_{3}{ }^{b}:=\left[\begin{array}{llllll}
1 & -1 & 1 & -1 & 0 & 0
\end{array}\right]^{\prime} \\
& \mathrm{E}_{4}{ }^{b}:=\left[\begin{array}{llllll}
C^{b} & -\varepsilon & C^{b} & C^{b} & 0 & 0
\end{array}\right]^{\prime} \\
& \mathrm{E}_{1}{ }^{g}:=\left[\begin{array}{llllll}
T^{g} & -\left(T^{g}+\varepsilon\right) & M^{g} & M^{g} & -M^{g} & -M^{g}
\end{array}\right]^{\prime} \\
& \mathrm{E}_{3}{ }^{g}(k):=\left[\begin{array}{llllll}
1 & -1 & c^{P}(k) & -c^{P}(k) & c^{S}(k) & -c^{S}(k)
\end{array}\right]^{\prime} \\
& \mathrm{E}_{4}{ }^{g}:=\left[\begin{array}{llllll}
T^{g} & -\varepsilon & M^{g} & M^{g} & 0 & 0
\end{array}\right]^{\prime}
\end{aligned}
$$

where $M^{g}:=\max _{k}\left(c^{P}(k), c^{S}(k)\right) \cdot T^{g}, \varepsilon$ is a small tolerance (typically the machine precision).

$$
\begin{aligned}
& \boldsymbol{F}(k):=\left[\begin{array}{cccccc}
1 & \underbrace{1 \ldots 1}_{N_{g}} & -1 & \underbrace{\ldots D_{i}^{c}(k) \ldots}_{N_{c}} & \underbrace{0 \ldots 0}_{N_{c h p}} & \underbrace{0 \ldots 0}_{N_{g}+2} \\
0 & \underbrace{0 \ldots 0}_{N_{g}} & 0 & \underbrace{\ldots 0 \ldots}_{N_{c}} & \underbrace{1 \ldots 1}_{N_{c h p}} & \underbrace{0 \ldots 0}_{N_{g}+2}
\end{array}\right] \\
& \boldsymbol{f}:=\left[\begin{array}{llll}
1 & \underbrace{-1 \ldots-1}_{N_{l}} & \underbrace{-1 \ldots-1}_{N_{c}} & \underbrace{0 \ldots 0}_{N_{q}} \\
0 & \underbrace{0 \ldots 0}_{N_{l}} & \underbrace{0 \ldots 0}_{N_{c}} & \underbrace{1 \ldots 1}_{N_{q}}
\end{array}\right]
\end{aligned}
$$

\section{References}

[1] R. Lasseter, P. Piagi, Microgrid: a conceptual solution, in: IEEE Annual Power Electron Specialists Conference, 2004, pp. 4285-4290.

[2] N. Hatziargyriou, H. Asano, R. Iravani, C. Marnay, Microgrids, in: IEEE Power \& Energy Magazine, 2008.

[3] Strategic deployment document for Europe electricity networks of the future, 2008 http://www.smartgrids.eu/.

[4] T. Ustun, C. Ozansoy, A. Zayegh, Recent developments in microgrids and example cases around the world. A review, Renew. Sustain. Energy Rev. 15 (8) (2011) 4030-4041.

[5] J. Ilic, M. Prica, S. Rabiei, J. Goellner, D. Wilson, C. Shih, R. Egidi, Technical and economic analysis of various power generation resources coupled with CAES systems, Tech. rep., National Energy Technology Laboratory, 2011, June, DOE/NETL-2011/1472.

[6] M. Hoffman, M. Kintner-Meyer, A. Sadovsky, J. DeSteese, Analysis tools for sizing and placement of energy storage for grid applications - a literature review, Tech. rep., Pacific Northwest National Laboratory (DOE/PNNL), Richland, WA, US, 2010, September. 
[7] R. O'Neill, T. Dautel, E. Krall, Recent ISO software enhancements and future software and modeling plans. Staff report, Tech. rep., Federal Energy Regulatory Commission, 2011, November.

[8] R. Sioshansi, R. O'Neill, S. Oren, Economic consequences of alternative solution methods for centralized unit commitment in day-ahead electricity markets, IEEE Trans. Power Syst. 23 (2) (2008) 344-352.

[9] W. Su, J. Wang, Energy management systems in microgrid operations, Electr. J. (2012).

10] F. Mohamed, H. Koivo, Multiobjective optimization using mesh adaptive direct search for power dispatch problem of microgrid, Int. J. Electr. Power Energy Syst. 42 (2012) 728-735.

[11] A. Chaouachi, R. Kamel, R. Andoulsi, K. Nagasaka, Multiobjective intelligent energy management for a microgrid, IEEE Trans. Ind. Electron. 60 (4) (2013) 1688-1699.

[12] S. Chen, H. Gooi, Jump and shift method for multi-objective optimization, IEEE Trans. Ind. Electron. 58 (10) (2011).

13] M. Salani, A. Giusti, G.D. Caro, A. Rizzoli, L. Gambardella, Lexicographic multi-objective optimization for the unit commitment problem and economic dispatch in a microgrid, in: IEEE - PES International Conference on Smart Grid Technology (ISGT), 2011, pp. 1-8.

[14] A. Takeuchi, T. Hayashi, Y. Nozaki, T. Shimakage, Optimal scheduling using metaheuristics for energy networks, IEEE Trans. Smart Grid 3 (2) (2012) 968-974.

[15] P. Stluka, D. Godbole, T. Samad, Energy management for buildings and microgrids, in: IEEE Conference on Decision and Control, Orlando, FL, USA, 2011.

16] M. Sechilariu, B.C. Wang, F. Locment, Supervision control for optimal energy cost management in DC microgrid: design and simulation, Int. J. Electr. Powe Energy Syst. 58 (2014) 140-149, http://dx.doi.org/10.1016/j.ijepes.2014.01. 018 http://www.sciencedirect.com/science/article/pii/S0142061514000313.

[17] M. Marzband, M. Ghadimi, A. Sumper, J.L. Dominguez-Garcia, Experimental validation of a real-time energy management system using multi-period gravitational search algorithm for microgrids in islanded mode, Appl. Energy 128 (2014) 164-174.

[18] L. Guo, W. Liu, J. Cai, B. Hong, C. Wang, A two-stage optimal planning and design method for combined cooling, heat and power microgrid system, Energy Convers. Manage. 74 (2013) 433-445, http://dx.doi.org/10.1016/j. enconman.2013.06.051 http://www.sciencedirect.com/science/article/pii/ S0196890413003634.

[19] E. Kuznetsova, Y.-F. Li, C. Ruiz, E. Zio, An integrated framework of agent-based modelling and robust optimization for microgrid energy management, Appl. Energy 129 (2014) 70-88.

[20] A. Khodaei, Microgrid optimal scheduling with multi-period islanding constraints, IEEE Trans. Power Syst. 29 (3) (2014) 1383-1392, http://dx.doi. org/10.1109/TPWRS.2013.2290006.

[21] M. Kaut, S.W. Wallace, Evaluation of scenario-generation methods for stochastic programming. http://edoc.huberlin.de/series/speps/2003-14/PDF/ 14.pdf.

[22] S. Mirkhani, Y. Saboohi, Stochastic modeling of the energy supply system with uncertain fuel price. A case of emerging technologies for distributed power generation, Appl. Energy 93 (2012) 668-674.

[23] R. Anderson, A. Boulanger, W. Powell, W. Scott, Adaptive stochastic control for the smart grid, Proc. IEEE 99 (6) (2011) 1098-1115.

[24] B. Shengrong, F. Yu, P. Liu, Stochastic unit commitment in smart grid communications, in: Computer Communications Workshops (INFOCOM WKSHPS), Shanghai, 2011, pp. 307-312.

[25] S. Kennedy, M. Marden, Reliability of islanded microgrids with stochastic generation and prioritized load, in: IEEE PowerTech, 2009, pp. 1-7.

[26] T. Niknam, R. Azizipanah-Abarghooee, M. Narimani, An efficient scenario-based stochastic programming framework for multi-objective optimal micro-grid operation, Appl. Energy 99 (2012) 455-470.

[27] Z. Wang, S. Member, B. Chen, J. Wang, S. Member, M.M. Begovic, C. Chen, Coordinated energy management of networked microgrids in distribution systems, IEEE Trans. Smart Grid PP (99) (2014) 1-9.

[28] A.E. Nezhad, M.S. Javadi, E. Rahimi, Applying augmented $\varepsilon$-constraint approach and lexicographic optimization to solve multi-objective hydrothermal generation scheduling considering the impacts of pumped-storage units, Int. J. Electr. Power Energy Syst. 55 (2014) 195-204.

[29] A. Zakariazadeh, S. Jadid, P. Siano, Stochastic multi-objective operational planning of smart distribution systems considering demand response programs, Electr. Power Syst. Res. 111 (2014) 156-168.

[30] S. Mohammadi, S. Soleymani, B. Mozafari, Scenario-based stochastic operation management of microgrid including wind, photovoltaic, micro-turbine, fuel cell and energy storage devices, Int. J. Electr. Power Energy Syst. 54 (2014) 525-535.

[31] M. Hemmati, N. Amjady, M. Ehsan, System modeling and optimization for islanded micro-grid using multi-cross learning-based chaotic differential evolution algorithm, Int. J. Electr. Power Energy Syst. 56 (2014) 349360.

[32] L. Ji, D. Niu, G. Huang, An inexact two-stage stochastic robust programming for residential micro-grid management-based on random demand, Energy 67 (2014) 186-199.

[33] G. Cardoso, M. Stadler, A. Siddiqui, N. DeForest, A. Barbosa-Póvoa, P. Ferrão, Microgrid reliability modeling and battery scheduling using stochastic linear programming, Electr. Power Syst. Res. 103 (2013) 61-69.
[34] A. Baziar, A. Kavousi-Fard, Considering uncertainty in the optimal energy management of renewable micro-grids including storage devices, Renew. Energy 59 (2013) 158-166.

[35] Y. Zhang, N. Gatsis, G. Giannakis, Robust energy management for microgrids with high-penetration renewables, IEEE Trans. Sustain. Energy 4 (4) (2013) 944-953.

[36] W. Su, J. Wang, J. Roh, Stochastic energy scheduling in microgrids with intermittent renewable energy resources, IEEE Trans. Smart Grid PP (99) (2013) 1-8.

[37] R.B. Hytowitz, K.W. Hedman, Managing solar uncertainty in microgrid systems with stochastic unit commitment, Electr. Power Syst. Res. 119 (2015) 111-118.

[38] E. Kuznetsova, C. Ruiz, Y.-F. Li, E. Zio, Analysis of robust optimization for decentralized microgrid energy management under uncertainty, Int. J. Electr. Power Energy Syst. 64 (2015) 815-832, http://dx.doi.org/10.1016/j.ijepes. 2014.07.064.

[39] H. Shayeghi, B. Sobhani, Integrated offering strategy for profit enhancement of distributed resources and demand response in microgrids considering system uncertainties, Energy Convers. Manage. 87 (2014) 765-777, http://dx. doi.org/10.1016/j.enconman.2014.07.068 http://www.sciencedirect.com/ science/article/pii/S0196890414007067.

[40] B. Otomega, A. Marinakis, M. Glavic, T.V. Cutsem, Model predictive control to alleviate thermal overloads, IEEE Trans. Power Syst. 22 (3) (2007) 1384-1385.

[41] P. Meibom, R. Barth, B. Hasche, H. Brand, C. Weber, M. O'Malley, Stochastic optimization model to study the operational impacts of high wind penetrations in Ireland, IEEE Trans. Power Syst. 26 (3) (2011) 1367-1379.

[42] A. Hooshmand, H. Malki, J. Mohammadpour, Power flow management of microgrid networks using model predictive control, Comput. Math. Appl. (2012).

[43] W. Qi, J. Liu, X. Chen, P. Christofides, Supervisory predictive control of standalone wind/solar energy generation systems, IEEE Trans. Control Syst. Technol. 19 (1) (2011) 199-207.

[44] Y. Xu, L. Xie, C. Singh, Optimal scheduling and operation of load aggregators with electric energy storage facing price and demand uncertainties, in: North American Power Symposium (NAPS), 2011, pp. 1-7.

[45] I. Prodan, E. Zio, A model predictive control framework for reliable microgrid energy management, Int. J. Electr. Power Energy Syst. 61 (2014) 399-409, http://dx.doi.org/10.1016/j.ijepes.2014.03.017 http://www.sciencedirect. com/science/article/pii/S0142061514001197.

[46] R. Palma-Behnke, C. Benavides, F. Lanas, B. Severino, L. Reyes, J. Llanos, D. Saez, A microgrid energy management system based on the rolling horizon strategy, IEEE Trans. Smart Grid 4 (2) (2013) 996-1006.

[47] W. Qi, J. Liu, P.D. Christofides, A distributed control framework for smart grid development: energy/water system optimal operation and electric grid integration, J. Process Control 21 (10) (2011) 1504-1516, Special Issue: Selected Papers From Two Joint $\{$ IFAC $\}$ Conferences: 9th International Symposium on Dynamics and Control of Process Systems and the 11th International Symposium on Computer Applications in Biotechnology, Leuven, Belgium, July 5-9, 2010. doi:10.1016/j.jprocont.2011.05.010. http:// www.sciencedirect.com/science/article/pii/S0959152411001077.

[48] A. Elaiw, X. Xia, A. Shehata, Application of model predictive control to optimal dynamic dispatch of generation with emission limitations, Electr. Power Syst. Res. 84 (1) (2012) 31-44.

[49] P. Patrinos, S. Trimboli, A. Bemporad, Stochastic MPC for real-time market-based optimal power dispatch, in: IEEE Conference on Decision and Control, Orlando, FL, USA, 2011.

[50] A. Hooshmand, M. Poursaeidi, J. Mohammadpour, H. Malki, K. Grigoriads, Stochastic model predictive control method for microgrid management, in: IEEE Innovative Smart Grid Technologies (ISGT), PES, 2012, pp. 1-7.

[51] A. Bidram, A. Davoudi, Hierarchical structure of microgrids control system, IEEE Trans. Smart Grid 3 (4) (2012) 1963-1976.

[52] J. Maciejowski, Predictive Control with Constraints, Prentice Hall, Harlow, UK, 2002.

[53] A. Richard, J. How, Mixed-integer programming for control, in: American Control Conference, vol. 4, Portland, OR, USA, 2005, pp. 2676-2683.

[54] A. Shapiro, D. Dentcheva, A. Ruszczyński, Lectures on Stochastic Programming: Modeling and Theory, MPS-SIAM Series on Optimization, Society for Industrial \& Applied Mathematics, USA, 2009.

[55] R. Marler, J. Arora, Survey of multi-objective optimization methods fo engineering, Struct. Multidiscip. Optim. 26 (6) (2004) 369-395.

[56] G. Nemhauser, L. Wolsey, Integer and Combinatorial Optimization, Wiley, 1988.

[57] C. Floudas, Nonlinear and Mixed-Integer Programming - Fundamentals and Applications, Oxford University Press, Oxford, UK, 1995.

[58] A. Bemporad, M. Morari, Control of systems integrating logic, dynamics, and constraints, Automatica 35 (3) (1999) 407-427.

[59] A. Parisio, L. Glielmo, Stochastic model predictive control for economic/environmental operation management of microgrids, in: 2013 European Control Conference (ECC), 2013, pp. 2014-2019.

[60] A. Parisio, E. Rikos, L. Glielmo, A model predictive control approach to microgrid operation optimization, IEEE Trans. Control Syst. Technol. 22 (5) (2014) 1813-1827.

[61] A. Parisio, L. Glielmo, Use of model predictive control for experimenta microgrid optimization, Appl. Energy 115 (2014) 37-46.

[62] Z. Wu, W. Gu, R. Wang, X. Yuan, W. Liu, Economic optimal schedule of CHP microgrid system using chance constrained programming and particle swarm 
G Model

JJPC 2028 1-14

14

A. Parisio et al. / Journal of Process Control $x x x$ (2016) $x x x-x x x$

1063

1064

1065

1067

1068

1069

1070

1072

1073

1074

1075

1076

1077

1078
1079

1080

1081

1082

1083

1085 optimization, in: IEEE Power and Energy Society General Meeting, Detroit, MI, USA, 2011.

[63] Catalog of CHP Technologies, Tech. rep., U.S. Environmental Protection Agency Combined Heat and Power Partnership, 2008, December.

[64] M. Carrión, J. Arroyo, A computationally efficient mixed-integer linear formulation for the thermal unit commitment problem, IEEE Trans. Power Syst. 21 (3) (2006) 1371-1378.

[65] A. Bemporad, Model predictive control of hybrid systems, in: Advanced Process Control Applications for Industry Workshop, Vancouver, British Columbia, 2007.

[66] M. Abide, Environmental/economic power dispatch using multiobjective evolutionary algorithms, IEEE Trans. Power Syst. 18 (4) (2003) 15291537.

[67] N. Sharma, P. Sharma, D. Irwin, P. Shenoy, Predicting solar generation from weather forecasts using machine learning, in: Proceedings of the Second IEEE International Conference on Smart Grid Communications (SmartGridComm), Brussels, Belgium, 2011.

[68] L. Yule, Y. Yafeng, G. Junjun, T. Chongli, Demand forecasting by using support vector machine, in: Third International Conference on Natural Computation, vol. 3, 2007, pp. 272-276.

[69] E. Osuna, R. Freund, F. Girosi, Support vector machines: training and applications, Tech. Rep. AIM-1602, CBCL-144, Computer Science and Artificial Intelligence Lab (CSAIL), Massachusetts Institute of Technology, 1997, March http://hdl.handle.net/1721.1/7290.
[70] W. Römisch, S. Vigerske, Recent progress in two-stage mixed-integer stochastic programming with applications to power production planning, in: S. Rebennack, P. Pardalos, M. Pereira, N. Iliadis (Eds.), Handbook of Power Systems I, Energy Systems, Springer, Berlin/Heidelberg, 2010, pp. 177-208.

[71] W. Zucchini, I. MacDonald, Hidden Markov Models for Time Series, MacDonald Chapman and Hall/CRC, 2009.

[72] H. Heitsch, W. Römisch, Scenario reduction algorithms in stochastic programming, J. Compute. Optim. Appl. 24 (2-3) (2003) 187-206.

[73] K. Bhattacharya, J. Zhong, Reactive power as an ancillary service, IEEE Power Eng. Rev. 21 (5) (2001) 64, http://dx.doi.org/10.1109/MPER.2001.4311391.

[74] E.S. Programme, Contract n XVII/4. 1031/P/99-159, EDUCOGEN The European Education Tool on Energy-Efficiency through the Use of Cogeneration, Tech. Rep., 2002, March.

[75] ILOG, CPLEX 12.0 Users Manual, 2010.

[76] K. Pelckmans, J. Suykens, T. VanGestel, J. DeBrabanter, L. Lukas, B. Homers, B. DeMoor, J. Vandewalle, LS-SVMlab: a MATLAB/C toolbox for Least Squares Support Vector Machines, 1998, ESAT-SCD-SISTA K.U. Leuven Kasteelpark Arenberg 10, B-3001 Leuven-Heverlee, Belgium.

[77] http://www.cs.ubc.ca/murphyk/Software/HMM/hmm.html.

[78] A. Bemporad, D. de la Peña, Multi-objective model predictive control, Automatic 45 (12) (2009) 2823-2830.

[79] E. Camacho, D. Ramirez, D. Limon, D. de la Peña, T. Alamo, Model predictive control techniques for hybrid systems, Annul. Rev. Control 34 (1) (2010) 21-31.
1086

1087

1088

1089

1090

1091

1092

1093

1095

1096

1097

1098

1099

1100

1101

1102

1103

1104

1106

1107

1108
1109

Please cite this article in press as: A. Parisio, et al., Stochastic model predictive control for economic/environmental operation managemont of microgrids: An experimental case study, J. Process Control (2016), http://dx.doi.org/10.1016/j.jprocont.2016.04.008 